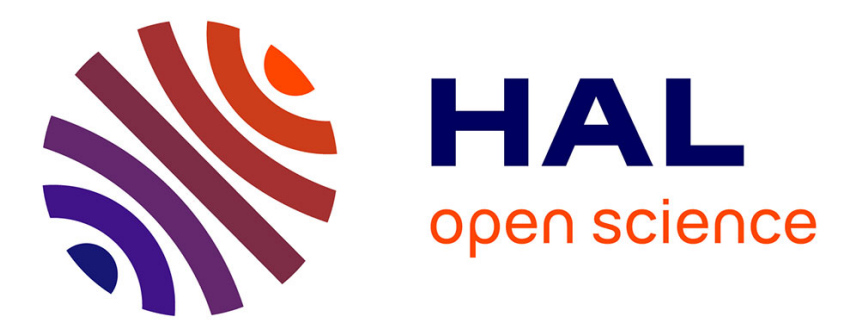

\title{
Singular integral operators with operator-valued kernels, and extrapolation of maximal regularity into rearrangement invariant Banach function spaces
}

Ralph Chill, Alberto Fiorenza

\section{- To cite this version:}

Ralph Chill, Alberto Fiorenza. Singular integral operators with operator-valued kernels, and extrapolation of maximal regularity into rearrangement invariant Banach function spaces. Journal of Evolution Equations, 2014, 14 (4), pp.795-828. 10.1007/s00028-014-0239-1 . hal-01275671

\section{HAL Id: hal-01275671 \\ https://hal.science/hal-01275671}

Submitted on 17 Feb 2016

HAL is a multi-disciplinary open access archive for the deposit and dissemination of scientific research documents, whether they are published or not. The documents may come from teaching and research institutions in France or abroad, or from public or private research centers.
L'archive ouverte pluridisciplinaire HAL, est destinée au dépôt et à la diffusion de documents scientifiques de niveau recherche, publiés ou non, émanant des établissements d'enseignement et de recherche français ou étrangers, des laboratoires publics ou privés. 


\title{
SINGULAR INTEGRAL OPERATORS WITH OPERATOR-VALUED KERNELS, AND EXTRAPOLATION OF MAXIMAL REGULARITY INTO REARRANGEMENT INVARIANT BANACH FUNCTION SPACES
}

\author{
RALPH CHILL AND ALBERTO FIORENZA
}

\begin{abstract}
АвstRact. We prove two extrapolation results for singular integral operators with operator-valued kernels and we apply these results in order to obtain the following extrapolation of $L^{p}$-maximal regularity: if an autonomous Cauchy problem on a Banach space has $L^{p}$-maximal regularity for some $p \in(1, \infty)$, then it has $\mathbb{E}_{w}$-maximal regularity for every rearrangement-invariant Banach function space $\mathbb{E}$ with Boyd indices $1<p_{\mathbb{E}} \leq q_{\mathbb{E}}<\infty$ and every Muckenhoupt weight $w \in A_{p_{\mathbb{E}}}$. We prove a similar result for non-autonomous Cauchy problems on the line.
\end{abstract}

\section{INTRODUCTION}

The theory of maximal regularity of abstract linear evolution equations has proved to be fundamental because of its applications to nonlinear problems. It is moreover a beautiful theory which combines results and techniques from Fourier analysis and abstract harmonic analysis on Banach spaces, from the theory of singular integral operators, from Banach space geometry, from operator theory and from variational analysis. In this article, by applying a recent extrapolation result of Curbera, García-Cuerva, Martell and Perez [17] (which goes back to an idea of Rubio de Francia [44]) and a Coifman-Fefferman inequality for Calderón-Zygmund operators with operator-valued kernels, we obtain the following statement (Corollary 7.4).

If $A$ is a linear, closed and densely defined operator on a Banach space $X$ and if the first order problem

$$
\dot{u}+A u=f \quad \text { on }(0, T), \quad u(0)=0,
$$

Date: March 6, 2014.

2010 Mathematics Subject Classification. Primary 42B20, 34G10; Secondary 47D06, 46E30.

Key words and phrases. singular integral operator, Calderón-Zygmund operator, CoifmanFefferman inequality, rearrangement invariant Banach function space, Boyd's interpolation theorem, nonautonomous Cauchy problem, maximal regularity, extrapolation, interpolation.

This paper started during the visit of the second author at the Paul Verlaine University of Metz, France. He wishes to thank for the support and warm hospitality. 
has $L^{p}$-maximal regularity for some $p \in(1, \infty)$, then it has $\mathbb{E}_{w}$-maximal regularity for every rearrangement-invariant Banach function space $\mathbb{E}$ with Boyd indices $1<p_{\mathbb{E}} \leq$ $q_{\mathbb{E}}<\infty$ and every Muckenhoupt weight $w \in A_{p_{\mathbb{E}}}$.

Let us recall that the above Cauchy problem has $L^{p}$-maximal regularity if for every $f \in L^{p}(0, T ; X)$ there exists a unique solution $u \in L^{p}(0, T ; X)$ such that $\dot{u}$ and $A u$ also belong to $L^{p}(0, T ; X)$. The notion of maximal regularity is defined similarly for non-autonomous problems, second order problems or evolutionary integral equations, the important requirement being that the unknown terms in the differential equation each have the same regularity as the given right-hand side.

In the definition of maximal regularity, the space $L^{p}$ can be replaced (and this was actually done in the literature) by other function spaces. We see three prominent types of spaces.

There is for example the space of continuous functions and, more generally, spaces of continuously differentiable functions. It is true that C-maximal regularity has been studied and holds in some situations, but we recall that the Cauchy problem with unbounded $A$ has $C$-maximal regularity only if the space $X$ contains $c_{0}$ [8]. Hence, $C$-maximal regularity never occurs if $A$ is an unbounded operator on a reflexive space (for example, an $L^{q}$ space).

Maximal regularity in spaces of Hölder continuous functions or in Besov spaces has been studied, too, and we recall that $C^{\alpha}$ - and $B_{p q}^{s}$-maximal regularity hold as soon as $-A$ generates an analytic $C_{0}$-semigroup on $X$. This follows from an abstract maximal regularity result in interpolation spaces for sums of sectorial operators [19].

Finally, one can study maximal regularity in spaces of measurable functions, for example in $L^{p}$ spaces ( $L^{p}$-maximal regularity), but also in weighted $L^{p}$ spaces, Orlicz spaces, Lorentz spaces etc. However, it seems that only the $L^{p}$ spaces and their weighted versions appear in the literature. For example, it is known that if the Cauchy problem has $L^{p}$-maximal regularity for some $p \in(1, \infty)$, then $-A$ generates an analytic $C_{0}$-semigroup [23]. The converse is true in Hilbert spaces [20], but it is not true in general [33]. This makes the theory of $L^{p}$-maximal regularity more involved and gave rise to the notion of $R$-boundedness of operator families and a characterization of $L^{p}$-maximal regularity on UMD Banach spaces (see [35], [4], [21] for accounts of the theory).

The above mentioned extrapolation result closes the gap between the existing theory of $L^{p}$-maximal regularity and the surprising absence of results in more general Banach function spaces of measurable functions. It extends two existing extrapolation results. The first one is well known, follows from the Marcinkiewicz interpolation theorem and states that if the Cauchy problem has $L^{p}$-maximal regularity for some $p \in(1, \infty)$, then it has $L^{p}$-maximal regularity for every $p \in(1, \infty)[45],[13],[29]$. The second one is more recent and states 
extrapolation of $L^{p}$-maximal regularity into $L_{w}^{p}$-maximal regularity for certain power weights $w$ [7], [43].

These extrapolation results are important with respect to solvability and regularity of the initial value problem

$$
\dot{u}+A u=0 \quad \text { on }(0, T), \quad u(0)=x,
$$

which in turn are important for the study of nonlinear problems. They imply for example precise $L_{w}^{p}$ regularity results when the initial values belong to the classical real interpolation spaces $(X, D(A))_{\theta, p}$ which may be defined as trace spaces of appropriate maximal regularity spaces. The $\mathbb{E}_{w}$-maximal regularity and associated maximal regularity spaces gives rise to a whole class of new trace spaces. The study of this class of trace spaces certainly deserves attention but is not the subject of this article.

The article is organized as follows. In Sections 2 and 3 we recall some background on Banach function spaces, the Hardy-Littlewood maximal operator and the Muckenhoupt weights. In Section 4 we recall the definition of singular integral operators and Calderón-Zygmund operators, and we state the main abstract extrapolation results including the Coifman-Fefferman inequality for Calderón-Zygmund operators with operator valued kernels. Sections 5 and 6 are devoted to the proofs of the abstract extrapolation results. Finally, in Section 7 we apply Theorem 4.3 in order to obtain the extrapolation result stated above. We actually also include the study of a nonautonomous problem there.

\section{Background from Banach Function Spaces Theory}

2.1. Scalar valued functions. Fix $n \geq 1$. In the following, unless otherwise specified, all our function spaces are spaces of functions defined on $\mathbb{R}^{n}$. Let $\mathcal{M}$ be the set of the Lebesgue measurable, complex valued functions on $\mathbb{R}^{n}$, and let $\mathcal{M}^{+}$be the subset of real valued, non-negative functions. For a measurable set $E \subseteq \mathbb{R}^{n}$, by $m(E)$ and $\chi_{E}$ we denote respectively the Lebesgue measure of $E$ and the characteristic function of $E$. A Banach function norm $\rho$ (see Bennett and Sharpley [10] for this and the next definitions, and details on the assertions appearing in the text below) is a mapping $\rho: \mathcal{M}^{+} \rightarrow[0, \infty]$ such that the following properties hold:

- $\rho(f)=0 \Leftrightarrow f=0$ almost everywhere,

- $\rho(f+g) \leq \rho(f)+\rho(g)$,

- $\rho(\lambda f)=\lambda \rho(f)$ for all $\lambda \geq 0$,

- if $0 \leq f \leq g$ almost everywhere, then $\rho(f) \leq \rho(g)$,

- if $f_{n} \nearrow f$ almost everywhere, then $\rho\left(f_{n}\right) \nearrow \rho(f)$,

- if $m(E)<\infty$, then $\rho\left(\chi_{E}\right)<\infty$

- if $m(E)<\infty$, then $\int_{E} f d x \leq C_{E} \rho(f)$ for some $C_{E}$ depending on $E$ and $\rho$, but independent of $f$. 
By means of a Banach function norm $\rho$, a function space $\mathbb{E}=\mathbb{E}(\rho)$ is defined:

$$
\mathbb{E}=\{f \in \mathcal{M}: \rho(|f|)<\infty\} .
$$

This space becomes a Banach space of functions on $\left(\mathbb{R}^{n}, d x\right)$ under the norm $\|f\|_{\mathbb{E}}=\rho(|f|)$. We call it Banach Function Space over $\left(\mathbb{R}^{n}, d x\right)$, briefly BFS (the underlying measure space is generally omitted). An analogous definition on other measure spaces holds, and, when used, the underlying measure space is indicated.

For a measurable function $f$ we define its distribution function $m_{f}$ by

$$
m_{f}(\lambda)=m\left(\left\{x \in \mathbb{R}^{n}:|f(x)|>\lambda\right\}\right) \quad(\lambda \geq 0),
$$

and we say that two measurable functions $f$ and $g$ are equimeasurable if they have the same distribution function, that is, $m_{f}=m_{g}$. A BFS $\mathbb{E}$ is rearrangement invariant (we write briefly r.i.BFS) if $\rho(f)=\rho(g)$ for every pair of functions in $\mathbb{E}$ which are equimeasurable. This means that the norm of a function $f$ depends only on the measure of the level sets of $|f|$.

The decreasing rearrangement of $f$ is the function $f^{*} \in \mathcal{M}^{+}\left(\mathbb{R}_{+}\right)$defined by

$$
f^{*}(t)=\inf \left\{\lambda \geq 0: m_{f}(\lambda) \leq t\right\} \quad(t \geq 0) .
$$

We use here the convention that $\inf \emptyset=\infty$. Since $f^{*}$ is defined starting from the distribution function of $f$, of course two equimeasurable functions have the same decreasing rearrangement. Moreover, $f$ and $f^{*}$ have the same distribution function, that is, $m_{f}=m_{f^{*}}$.

By Luxemburg's representation theorem [10, p.62], every r.i.BFS $\mathbb{E}$ has a representation on the measure space $([0, \infty[, d t)$, that is, there exists a r.i.BFS $\overline{\mathbb{E}}$ over $\left(\left[0, \infty[, d t)\right.\right.$ such that $f \in \mathbb{E}$ if and only if $f^{*} \in \overline{\mathbb{E}}$, and in this case $\|f\|_{\mathbb{E}}=\left\|f^{*}\right\|_{\overline{\mathbb{E}}}$.

As in Curbera, García-Cuerva, Martell, and Pérez [17], we define the weighted versions of the r.i.BFS in the following way. Let $w$ be a weight, that is, a positive, locally integrable function on $\mathbb{R}^{n}$. For any measurable set $E \subseteq \mathbb{R}^{n}$, we set $w(E)=\int_{E} w(x) d x$. For any $f \in \mathcal{M}$, the distribution function and the decreasing rearrangement with respect to $w$ are respectively defined by

$$
\begin{aligned}
& w_{f}(\lambda)=w\left(\left\{x \in \mathbb{R}^{n}:|f(x)|>\lambda\right\}\right) \quad(\lambda \geq 0) \text { and } \\
& f_{w}^{*}(t)=\inf \left\{\lambda \geq 0: w_{f}(\lambda) \leq t\right\} \quad(t \geq 0) .
\end{aligned}
$$

It can be proven that the distribution function is nonnegative, nonincreasing and right-continuous (see, for example, [10, Proposition 1.3, p.37]). The BFS $\mathbb{E}_{w}$ is the r.i.BFS over $\left(\mathbb{R}^{n}, w(x) d x\right)$ defined by

$$
\mathbb{E}_{w}=\left\{f \in \mathcal{M}:\left\|f_{w}^{*}\right\|_{\overline{\mathbb{E}}}<\infty\right\},
$$

and its norm is $\|f\|_{\mathbb{E}_{w}}=\left\|f_{w}^{*}\right\|_{\overline{\mathbb{E}}}$. 
Let us now define the Boyd indices of a r.i.BFS. Given a r.i.BFS $\mathbb{E}$, the dilation operator $D_{t}: \overline{\mathbb{E}} \rightarrow \overline{\mathbb{E}}$ is the bounded linear operator defined by

$$
D_{t} f(s)=f(s / t), \quad 0<t<\infty, \quad f \in \overline{\mathbb{E}} \text {. }
$$

Set $h_{\mathbb{E}}(t)=\left\|D_{t}\right\|_{\mathcal{L}(\overline{\mathbb{E}})}$, where $\mathcal{L}(\overline{\mathbb{E}})$ denotes the space of bounded linear operators on $\overline{\mathbb{E}}$. The lower and upper Boyd indices are defined respectively by

$$
\begin{aligned}
& p_{\mathbb{E}}=\lim _{t \rightarrow \infty} \frac{\log t}{\log h_{\mathbb{E}}(t)}=\sup _{1<t<\infty} \frac{\log t}{\log h_{\mathbb{E}}(t)} \quad \text { and } \\
& q_{\mathbb{E}}=\lim _{t \rightarrow 0+} \frac{\log t}{\log h_{\mathbb{E}}(t)}=\inf _{0<t<1} \frac{\log t}{\log h_{\mathbb{E}}(t)} .
\end{aligned}
$$

One always has $1 \leq p_{\mathbb{E}} \leq q_{\mathbb{E}} \leq \infty$ (see for example [10, Proposition 5.13, p.149], where the Boyd indices are defined as the reciprocals with respect to our definitions).

Here are some examples of the definitions given above. To simplify the notation, in every example the function norm is indicated with the same symbol $\rho$. We stress that it is not our purpose to give the definitions in their utmost generality; we restrict ourselves to the spaces that can be considered in the rest of the paper as special cases of BFS.

Example 2.1 (Lebesgue spaces). Let $1 \leq p \leq \infty$, and set

$$
\rho(f)=\left\{\begin{array}{ll}
\left(\int_{\mathbb{R}^{n}} f(x)^{p} d x\right)^{1 / p} & \text { if } 1 \leq p<\infty, \\
\underset{\mathbb{R}^{n}}{\operatorname{ess} \sup f} & \text { if } p=\infty,
\end{array} \quad f \in \mathcal{M}^{+} .\right.
$$

The resulting r.i.BFS space is the classical Lebesgue space $L^{p}=L^{p}\left(\mathbb{R}^{n}\right)$, whose upper and lower Boyd indices coincide and are equal to $p$. Since $f$ and $f^{*}$ are equimeasurable, and since for $1 \leq p<\infty$

$$
\int_{\mathbb{R}^{n}}|f(x)|^{p} d x=p \int_{0}^{\infty} \lambda^{p-1} m_{f}(\lambda) d \lambda=p \int_{0}^{\infty} \lambda^{p-1} m_{f^{*}}(\lambda) d \lambda=\int_{0}^{\infty} f^{*}(t)^{p} d t,
$$

we have $\overline{\mathbb{E}}=L^{p}(0, \infty)$. The same inequality holds trivially when $p=\infty$.

Example 2.2 (Intersections and sums of Lebesgue spaces). If $1 \leq p, q \leq \infty$, then the intersection $L^{p} \cap L^{q}$ and the sum $L^{p}+L^{q}$, both equipped with the natural norms, are r.i.BFS and $\left(L^{p} \cap L^{q}\right)^{\prime}=L^{p^{\prime}}+L^{q^{\prime}},\left(L^{p}+L^{q}\right)^{\prime}=L^{p^{\prime}} \cap L^{q^{\prime}}$ (see [10, Example 6 , p.175]). The Boyd indices of $\mathbb{E}=L^{p} \cap L^{q}, p \leq q$, are $p_{\mathbb{E}}=p, q_{\mathbb{E}}=q$ (see [10, Example 13, p.177]). 
Example 2.3 (Weighted Lebesgue spaces). Let $1 \leq p<\infty$, let $w$ be a weight defined on $\mathbb{R}^{n}$, and set

$$
\rho_{1}(f)=\left(\int_{\mathbb{R}^{n}} f(x)^{p} w(x) d x\right)^{1 / p} \quad \text { for } f \in \mathcal{M}^{+} .
$$

The resulting BFS is the classical weighted Lebesgue space $L^{p}\left(\mathbb{R}^{n} ; w(x) d x\right)$, which is not rearrangement invariant unless $w$ is constant.

On the other hand, the abstract construction of weighted BFS spaces given above leads to the spaces defined by

$$
\rho_{2}(f)=\left(\int_{0}^{\infty} f_{w}^{*}(t)^{p} d t\right)^{1 / p} \quad \text { for } f \in \mathcal{M}^{+} .
$$

The resulting BFS space $\mathbb{E}$ is the space to be denoted by the symbol $L_{w}^{p}$, in accordance with the notation of $\mathbb{E}_{w}$.

Observe, however, that the two spaces $L^{p}\left(\mathbb{R}^{n} ; w(x) d x\right)$ and $L_{w}^{p}$ coincide. In fact,

$$
\int_{\mathbb{R}^{n}} f(x)^{p} w(x) d x=\int_{0}^{\infty} \lambda^{p} w_{f}(\lambda) d \lambda
$$

and

$$
\int_{0}^{\infty} f_{w}^{*}(t)^{p} d t=\int_{0}^{\infty} \lambda^{p} m_{f_{w}^{*}}(\lambda) d \lambda .
$$

Therefore, the asserted equality can be proved from the following equality, which is true for all nonincreasing, right-continuous functions $g$ on $[0, \infty[$,

$$
\{t \geq 0: \inf \{\sigma \geq 0: g(\sigma) \leq t\}>\lambda\}=[0, g(\lambda)[
$$

and which has to be applied with $g(\lambda)=w_{f}(\lambda)$, and then considering the Lebesgue measure of both sides. Equality (2.1) is simple: from the one hand, the inclusion " $\subseteq$ " follows from the fact that the inequality inf $\{\sigma \geq 0: g(\sigma) \leq t\}>\lambda$ implies $g(\lambda)>t$. On the other hand, every $t \in[0, g(\lambda)[$ is such that for some $\epsilon>0$ one has $t<g(\lambda)-\epsilon$, and therefore

$$
g(\sigma) \leq t \Rightarrow g(\sigma) \leq g(\lambda)-\epsilon,
$$

so that

$$
\{\sigma \geq 0: g(\sigma) \leq t\} \subseteq\{\sigma \geq 0: g(\sigma) \leq g(\lambda)-\epsilon\}
$$

and

$$
\inf \{\sigma \geq 0: g(\sigma) \leq t\} \geq \inf \{\sigma \geq 0: g(\sigma) \leq g(\lambda)-\epsilon\}>\lambda .
$$

Notice that the assumptions on $g$ were used in the last inequality. The equality above tells that the weighted Lebesgue spaces could be considered as rearrangement invariant, if equimeasurability is understood with respect to the measure induced by the weight $w$. 
We remark that for Banach Function Spaces on $((0, \infty), d t)$ the equality above, with a different notation, has been proved in Maligranda [38], where the equality corresponding to (2.1) goes back to Łojasiewicz [36].

Example 2.4 (Orlicz spaces). Let $\Phi:[0, \infty[\rightarrow[0, \infty[$ be strictly increasing, continuous, convex, such that $\Phi(0)=0, \lim _{s \rightarrow \infty} \Phi(s)=+\infty$, and set

$$
\rho(f)=\inf \left\{\lambda>0: \int_{\mathbb{R}^{n}} \Phi\left(\frac{f(x)}{\lambda}\right) d x \leq 1\right\} \quad \text { for } f \in \mathcal{M}^{+} .
$$

The resulting r.i.BFS space $\mathbb{E}$ is the classical Orlicz space $L^{\Phi}$, whose upper and lower Boyd indices can be computed by:

$$
p_{\mathbb{E}}=\lim _{t \rightarrow \infty} \frac{\log t}{\log g(t)} \quad \text { and } \quad q_{\mathbb{E}}=\lim _{t \rightarrow 0+} \frac{\log t}{\log g(t)},
$$

where $g$ is defined by

$$
g(t)=\limsup _{s \rightarrow \infty} \frac{\Phi^{-1}(s)}{\Phi^{-1}(s / t)} .
$$

Let us mention here that under quite general assumptions on $\Phi$, much easier formulas to compute the indices of Orlicz spaces can be found in Fiorenza and $\operatorname{Krbec}[25,26]$. One has $\overline{\mathbb{E}}=L^{\Phi}(0, \infty)$. When $\Phi(t)=t^{p}, 1 \leq p<\infty$, then the Orlicz space $L^{\Phi}$ reduces to the Lebesgue space $L^{p}$. When $\Phi(t)=t^{p}\left(1+\log ^{+} t\right)^{\alpha}$, $1 \leq p<\infty, \alpha \geq 0$, the Orlicz space reduces to the so-called Zygmund space $L^{p} \log ^{\alpha} L$. In this case $p_{\mathbb{E}}=q_{\mathbb{E}}=p$.

Example 2.5 (Weighted Orlicz spaces). Let $\Phi:[0, \infty[\rightarrow[0, \infty[$ be strictly increasing, continuous, convex, such that $\Phi(0)=0, \lim _{s \rightarrow \infty} \Phi(s)=+\infty$, and let $w$ be a weight. The functional

$$
\rho_{1}(f)=\inf \left\{\lambda>0: \int_{\mathbb{R}^{n}} \Phi\left(\frac{f(x)}{\lambda}\right) w(x) d x \leq 1\right\}, \quad f \in \mathcal{M}^{+},
$$

defines a Banach function norm. The resulting BFS is the classical weighted Orlicz space $L^{\Phi}\left(\mathbb{R}^{n} ; w(x) d x\right)$, which, as in the case of Lebesgue spaces, is not rearrangement invariant unless $w$ is constant.

By means of identity (2.1), similarly as before it is possible to show that the definition above is equivalent to that one coming from the functional

$$
\rho_{2}(f)=\inf \left\{\lambda>0: \int_{0}^{\infty} \Phi\left(\frac{\left.f_{w}^{*}(t)\right)}{\lambda}\right) d t \leq 1\right\}, \quad f \in \mathcal{M}^{+} .
$$


Example 2.6 (Lorentz spaces). Let $1<p \leq \infty$ and $1 \leq q \leq \infty$. The Lorentz spaces $L^{p, q}$ are defined by the function norm

$$
\rho(f)= \begin{cases}\left(\int_{0}^{\infty} s^{q / p} f^{* *}(s)^{q} \frac{d s}{s}\right)^{1 / q} & \text { if } q<\infty, \\ \sup _{s>0} s^{1 / p} f^{* *}(s) & \text { if } q=\infty .\end{cases}
$$

Here, $f^{* *}(s)=\frac{1}{s} \int_{0}^{s} f^{*}(r) d r$. We note that $f \in L^{p, q}$ if and only if

$$
\rho_{1}(f)= \begin{cases}\left(\int_{0}^{\infty} s^{q / p} f^{*}(s)^{q} \frac{d s}{s}\right)^{1 / q} & \text { if } q<\infty, \\ \sup _{s>0} s^{1 / p} f^{*}(s) & \text { if } q=\infty,\end{cases}
$$

is finite [10, Lemma 4.5, p. 216]. More precisely, the functions $\rho$ and $\rho_{1}$ are equivalent; we use this equivalence in Section 6 below. If $1 \leq q \leq p<\infty$, then $\rho_{1}$ is in fact a Banach function norm, too, but it is not a Banach function norm in general (see [18]). The space $L^{p, q}$ has both Boyd indices equal to $p$ (see, for example, [10, Theorem 4.3, p.218]). See also Ziemer [50] for further details on Lorentz spaces.

2.2. Banach space valued functions. Given a complex Banach space $X$ with norm $|\cdot|_{X}$, we let $\mathcal{M}(X)$ be the set of all (strongly) measurable (in the sense of [32, Definition 3.5.4]) functions $f: \mathbb{R}^{n} \rightarrow X$. Given a BFS $\mathbb{E}$, we define its variant for Banach space valued functions by

$$
\mathbb{E}(X)=\left\{f \in \mathcal{M}(X):|f(\cdot)|_{X} \in \mathbb{E}\right\} .
$$

When equipped with the norm

$$
\|f\|_{\mathbb{E}(X)}=\left\||f(\cdot)|_{X}\right\|_{\mathbb{E}}=\rho\left(|f|_{X}\right),
$$

the space $\mathbb{E}(X)$ is a Banach space. Therefore, we can still refer to such spaces as BFS. Clearly, $\mathbb{E}=\mathbb{E}(\mathbb{C})$. All the notions given in the first part of this section can be extended in a natural way to this more general context. For example, here is the definition of the distribution function (we do not use a different symbol for sake of simplicity):

$$
w_{f}(\lambda)=w\left(\left\{x \in \mathbb{R}^{n}:|f(x)|_{X}>\lambda\right\}\right) \quad(\lambda \geq 0) .
$$

Notice that the notions of decreasing rearrangement, r.i.BFS, Boyd indices remain the same with the same symbols. All the standard examples of BFS should be modified accordingly: for instance, the Banach valued weighted Orlicz spaces (which contain, as special cases, the Banach valued Lebesgue, weighted and unweighted, spaces) are normed by using the Banach function 
norm

$$
\rho(f)=\inf \left\{\lambda>0: \int_{\mathbb{R}^{n}} \Phi\left(\frac{|f(x)|_{X}}{\lambda}\right) w(x) d x \leq 1\right\} .
$$

3. BACKGROUND ON THE BOUNDEDNESS OF THE MAXIMAL OPERATOR AND

\section{MuCKENHOUPT WEIGHTS}

Let $X$ be a complex Banach space with norm $|\cdot|_{X}$. In accordance with the notation of the preceding section, we write $L^{p}(X)=L^{p}\left(\mathbb{R}^{n} ; X\right)$ and if $X=\mathbb{C}$, then we simply write $L^{p}$. For $f \in L_{\text {loc }}^{1}(X)$, we consider the Hardy-Littlewood maximal operator

$$
M f(x)=\sup _{Q \ni x} \frac{1}{|Q|} \int_{Q}|f(y)|_{X} d y \quad\left(x \in \mathbb{R}^{n}\right),
$$

where $Q \subseteq \mathbb{R}^{n}$ varies among the cubes with sides parallel to the coordinate axes.

The main well known property of the operator $M$ is its boundedness from $L^{p}(X)$ into $L^{p}$ if $1<p \leq \infty$ [46, Theorem 1, p.5]. Strictly speaking, this result is only proved for $X=\mathbb{C}$, but, by definition,

$$
M f=M|f(\cdot)|_{X} \quad \text { and } \quad\|f\|_{L^{p}(X)}=\left\||f(\cdot)|_{X}\right\|_{L^{p}}
$$

for every $f \in L^{p}(X)$ and the boundedness of $M$ follows from the scalar case. The boundedness of the maximal operator on more general Banach Function Spaces has been established in the following classical result by Lorentz and Shimogaki (see [10, Theorem 5.17, p.154]). This result, like the previous one, was proved for scalar functions only, but it immediately extends to Banach space valued functions by the same observation as above.

Theorem 3.1. Let $\mathbb{E}$ be a r.i.BFS. Then the maximal operator is bounded from $\mathbb{E}(X)$ into $\mathbb{E}$ if and only if $p_{\mathbb{E}}>1$.

The boundedness of the maximal operator in weighted Lebesgue spaces is the celebrated theorem of Muckenhoupt (see Coifman and Fefferman [14], Stein [47, Theorem 1, p.201]; here again, we write its formulation for Banach space valued functions), which states that if $w$ is a weight, then the inequality

$$
\int_{\mathbb{R}^{n}} M f(x)^{p} w(x) d x \leq C \int_{\mathbb{R}^{n}}|f(x)|_{X}^{p} w(x) d x
$$

holds for all $f \in L_{w}^{p}(X), 1<p<\infty$, if and only if $w$ belongs to the so-called Muckenhoupt class $A_{p}$ (we say that $w$ is an $A_{p}$ weight, and we write $w \in A_{p}$ ), that is, the class of all weights such that

$$
[w]_{A_{p}}=\sup _{Q}\left(\frac{1}{|Q|} \int_{Q} w(x) d x\right)\left(\frac{1}{|Q|} \int_{Q} w(x)^{-1 /(p-1)} d x\right)^{p-1}<\infty .
$$


Here $Q$ varies among all cubes $Q \subseteq \mathbb{R}^{n}$ with sides parallel to the coordinate axis. The Muckenhoupt class is defined also when $p=1$ and $p=\infty$. The weight $w$ belongs to $A_{1}$ if $M w(x) \leq C w(x)$, while the class $A_{\infty}$ is defined as the union of all $A_{p}$ classes with $p \geq 1$, and

$$
[w]_{A_{\infty}}=\inf _{p \geq 1}[w]_{A_{p}}=\lim _{p \rightarrow \infty}[w]_{A_{p}} .
$$

The first examples of $A_{p}$ weights are the powers, namely, $w(x)=|x|^{\alpha} \in A_{p}$ if and only if $-n<\alpha<n(p-1)$ (see for example [47, 1.5, p.196 or 6.4, p.218])

Remark 3.2. In dimension $n=1$, if we write the exponent $\alpha$ as $\alpha=p(1-\mu)$, this means that $w$ is an $A_{p}$ weight if and only if $1 / p<\mu<1+1 / p$.

\section{Singular integral operators on spaces of Banach space Valued FUNCTIONS}

Let $X$ and $Y$ be two complex Banach spaces with respective norms $|\cdot|_{X}$ and $|\cdot|_{\gamma}$. We denote by $\mathcal{L}(X, Y)$ the space of all bounded linear operators from $X$ into $Y$, and by $|\cdot|_{\mathcal{L}(X, Y)}$ the usual operator norm on this space.

Fix $1<p<\infty$ and $n \geq 1$. Throughout the following,

$T$ is a bounded linear operator from $L^{p}(X)$ into $L^{p}(Y)$.

Moreover, we assume that there exists a kernel $K \in L_{\text {loc }}^{1}\left(\mathbb{R}^{n} \times \mathbb{R}^{n} \backslash \Delta ; \mathcal{L}(X, Y)\right)$ (here $\Delta$ is the diagonal of $\mathbb{R}^{n} \times \mathbb{R}^{n}$, that is, $\Delta=\left\{(x, x): x \in \mathbb{R}^{n}\right\}$ ) such that

$$
\begin{aligned}
& T f(x)=\int_{\mathbb{R}^{n}} K(x, y) f(y) d y \\
& \text { for every } f \in C_{c}\left(\mathbb{R}^{n} ; X\right) \text { and almost every } x \notin \operatorname{supp} f .
\end{aligned}
$$

An operator satisfying (4.1) and (4.2) is in the following called a singular integral operator. Of course, by a standard approximation argument, we can use the equality (4.2) also for $L^{p}$ functions with compact support.

We consider in the following two types of conditions on the kernel $K$. We say that the kernel $K$ is a standard kernel (see Duoandikoetxea [24], but this terminology goes back to Coifman and Meyer [15]) if there exist constants $C_{K} \geq 0, \delta>0$ such that

$$
\begin{aligned}
& |K(x, y)|_{\mathcal{L}(X, Y)} \leq \frac{C_{K}}{|x-y|^{n}}, \\
& \left|K(x, y)-K\left(x, y^{\prime}\right)\right|_{\mathcal{L}(X, Y)} \leq C_{K} \frac{\left|y-y^{\prime}\right|^{\delta}}{|x-y|^{n+\delta}} \quad \text { if }\left|y-y^{\prime}\right| \leq \frac{1}{2}|x-y|, \\
& \left|K(x, y)-K\left(x^{\prime}, y\right)\right|_{\mathcal{L}(X, Y)} \leq C_{K} \frac{\left|x-x^{\prime}\right|^{\delta}}{|x-y|^{n+\delta}} \quad \text { if }\left|x-x^{\prime}\right| \leq \frac{1}{2}|x-y| .
\end{aligned}
$$


We say that $T$ is a (generalized) Calderón-Zygmund operator if $T$ is a singular integral operator with standard kernel. This definition differs slightly from the literature in the sense that only translation-invariant kernels are considered (see below), and the boundedness of $T$ on $L^{p}$ is replaced by the condition that the Fourier transform of the kernel belongs to $L^{\infty}$. In fact, if the Fourier transform of the kernel belongs to $L^{\infty}$ and if $X=Y=\mathbb{C}$, then the associated singular integral operator is bounded on $L^{2}$. This is no longer true in general for arbitrary Banach spaces, even if the kernel is scalar valued. In the general setting it is appropriate to assume boundedness of $T$ rather than an additional condition on the kernel.

We say that the kernel $K$ satisfies Hörmander's integral conditions, if there exists a constant $C_{K} \geq 0$ such that

$$
\begin{aligned}
& \sup _{Q} \sup _{y, y^{\prime} \in Q} \int_{Q_{2}^{c}}\left|K(x, y)-K\left(x, y^{\prime}\right)\right|_{\mathcal{L}(X, Y)} d x \leq C_{K} \quad \text { and } \\
& \sup \sup _{Q} \int_{x, x^{\prime} \in Q}\left|K(x, y)-K\left(x^{\prime}, y\right)\right|_{\mathcal{L}(X, Y)} d y \leq C_{K},
\end{aligned}
$$

where $Q$ varies among the cubes with sides parallel to the coordinate axes, and where we have denoted by $Q_{2}$ the double cube which has the same center as $Q$ but whose sides are twice as long as those of $Q$, and by $Q_{2}^{c}$ the complement of $Q_{2}$ in $\mathbb{R}^{n}$. It is easy to prove that every standard kernel satisfies Hörmander's integral conditions, but that the converse is not true.

The classical example of a Calderón-Zygmund operator on $L^{p}=L^{p}(\mathbb{R})(1<$ $p<\infty)$ is the Hilbert transform, given by the kernel $K(x, y)=\frac{1}{x-y}$ :

$$
H f(x)=\int_{\mathbb{R}} \frac{1}{x-y} f(y) d y ;
$$

[10, Theorem 4.9, p.139]. However, note that on $L^{p}(X)=L^{p}(\mathbb{R} ; X)$ the Hilbert transform is bounded if and only if $X$ is a UMD space [12], [11]. The boundedness of the Hilbert transform in the framework of the theory of Banach Function Spaces has been established in the following classical result of Boyd (see [10, Theorem 5.18, p.154]).

Theorem 4.1. Let $\mathbb{E}$ be a r.i.BFS. Then the Hilbert transform is bounded on $\mathbb{E}$ if and only if $1<p_{\mathbb{E}} \leq q_{\mathbb{E}}<\infty$.

For general singular integral operators, we have the following two extrapolation results which are the main results of this section. 
Theorem 4.2. Fix $1<p<\infty$. Let $T$ be a bounded singular integral operator from $L^{p}(X)$ into $L^{p}(Y)$.

(i) If the associated kernel satisfies the first Hörmander integral condition (4.6), then, for every r.i.BFS $\mathbb{E}$ satisfying $1<p_{\mathbb{E}} \leq q_{\mathbb{E}}<p$, there exists a constant $C \geq 0$ such that

$$
\|T f\|_{\mathbb{E}(Y)} \leq C\|f\|_{\mathbb{E}(X)} \quad \text { for every } f \in L^{p} \cap \mathbb{E}(X) .
$$

(ii) If the associated kernel satisfies Hörmander's integral conditions, then, for every r.i.BFS $\mathbb{E}$ satisfying $1<p_{\mathbb{E}} \leq q_{\mathbb{E}}<\infty$, there exists a constant $C \geq 0$ such that

$$
\|T f\|_{\mathbb{E}(Y)} \leq C\|f\|_{\mathbb{E}(X)} \quad \text { for every } f \in L^{p} \cap \mathbb{E}(X) .
$$

Under stronger assumptions on the kernel we have the following stronger result which is a variant (in fact, a special case) for Banach space valued functions of a result of [17, Theorems 2.2, 2.3].

Theorem 4.3. Let $T$ be a Calderón-Zygmund operator, let $M$ be the Hardy-Littlewood maximal function, and let $\mathbb{E}$ be a r.i.BFS.

(i) Suppose that $q_{\mathbb{E}}<\infty$. Then for every Muckenhoupt weight $w \in A_{\infty}$ there is a constant $C \geq 0$ depending only on $\mathbb{E}$ and $[w]_{A_{\infty}}$ such that

$$
\|T f\|_{\mathbb{E}_{w}(Y)} \leq C\|M f\|_{\mathbb{E}_{w}}
$$

for every $f$ such that the left-hand side is finite.

(ii) Suppose that $1<p_{\mathbb{E}} \leq \infty$. Then for every Muckenhoupt weight $w \in A_{\infty}$ there is a constant $C \geq 0$ depending only on $\mathbb{E}$ and $[w]_{A_{\infty}}$ such that

$$
\|M f\|_{\mathbb{E}_{w}} \leq C\|f\|_{\mathbb{E}_{w}(X)}
$$

for every $f$ such that the left-hand side is finite.

(iii) Suppose that $1<p_{\mathbb{E}} \leq q_{\mathbb{E}}<\infty$. Then for every Muckenhoupt weight $w \in A_{p_{\mathbb{E}}}$ there is a constant $C \geq 0$ depending only on $\mathbb{E}$ and $[w]_{A_{p_{\mathbb{E}}}}$ such that

$$
\|T f\|_{\mathbb{E}_{w}(Y)} \leq C\|f\|_{\mathbb{E}_{w}(X)}
$$

for every $f$ such that the left-hand side is finite. In particular, $T$ is bounded from $\mathbb{E}_{w}(X)$ into $\mathbb{E}_{w}(Y)$.

We point out that Theorem 4.3 (iii) is in general false for singular integral operators for which the kernel satisfies only the Hörmander integral conditions; see [39, Corollary 3.4]. Therefore, compared with Theorem 4.2, a stronger condition (here: the standard condition) is necessary if one considers weighted estimates.

The proof of Theorem 4.2 is carried out in Section 5. It is based on a generalization of the Marcinkiewicz interpolation theorem and from this point of view perhaps classical in the theory of singular integral operators. 
The proof of Theorem 4.3 (see Section 6) is more surprising, even in the scalar valued case. It is based on two extrapolation results.

The first one is in the case of scalar valued functions and a slightly different definition of Calderon-Zygmund operators due to Coifman and Fefferman [14]. It is contained in [28, Corollary 2.10], where an optimal dependence on $[w]_{A^{p}}$ appears. However, in Section 6, we propose, by completeness, all details of a proof which, even if it is not a direct consequence of the original theorem, essentially follows the lines of [14] and [46].

Theorem 4.4. Let $T$ be a Calderón-Zygmund operator from $L^{p}(X)$ into $L^{p}(Y)(1<$ $p<\infty)$. Then for every Muckenhoupt weight $w \in A_{p}$ there is a constant $C$ depending only on $p$ and $[w]_{A_{p}}$ such that

$$
\|T f\|_{L_{w}^{p}(Y)} \leq C\|M f\|_{L_{w}^{p}} \quad \text { for every } f \in L^{p} \cap L_{w}^{p}(X) .
$$

The second extrapolation result is the following recent generalization of an extrapolation theorem of Rubio de Francia [44] (compare also with [16, Theorem 4.10]).

Theorem 4.5 (Curbera et al., [17], Theorem 2.1 and Remark 2.5). Let $0<p_{0}<\infty$ and $\mathcal{F} \subseteq \mathcal{M}^{+} \times \mathcal{M}^{+}$be a family of couples of non-negative functions such that for every $w \in A_{\infty}$ there exists $C_{w} \geq 0$ such that

$$
\int_{\mathbb{R}^{n}} f(x)^{p_{0}} w(x) d x \leq C_{w} \int_{\mathbb{R}^{n}} g(x)^{p_{0}} w(x) d x \quad \text { for every }(f, g) \in \mathcal{F} .
$$

Let $\mathbb{E}$ be a r.i.BFS such that $q_{\mathbb{E}}<\infty$. Then for all $w \in A_{\infty}$ we have

$$
\|f\|_{\mathbb{E}_{w}(X)} \leq C\|g\|_{\mathbb{E}_{w}(X)} \text { for every }(f, g) \in \mathcal{F} .
$$

\section{Proof of Theorem 4.2}

The following two propositions are in the spirit [9, Theorem 2], where translation-invariant kernels were considered.

Proposition 5.1. Let $T$ be a singular integral operator from $L^{p}(X)$ into $L^{p}(Y)$. Assume that $K$ satisfies the first Hörmander integral condition (4.6). Then the operator $T$ is weak $(1,1)$ in the sense that there exists a constant $C \geq 0$ such that for every $\alpha>0$ and every $f \in L^{p} \cap L^{1}(X)$ one has

$$
m\left(\left\{x \in \mathbb{R}^{n}:|T f(x)|_{Y}>\alpha\right\}\right) \leq \frac{C}{\alpha} \int_{\mathbb{R}^{n}}|f(x)|_{X} d x .
$$

Proof. Fix $\alpha>0$ and $f \in L^{p} \cap L^{1}(X)$. Applying the corollary of [46, Theorem 4, Chapter I.3.4] to the function $|f(\cdot)|_{X}$, we obtain a decomposition $\mathbb{R}^{n}=F \cup \Omega$, 
$F \cap \Omega=\emptyset$, such that

$$
\begin{aligned}
& |f(x)|_{X} \leq \alpha \text { for almost every } x \in F, \\
& \Omega=\bigcup_{j} Q_{j} \text { for cubes } Q_{j} \text { such that } m\left(Q_{j} \cap Q_{k}\right)=0 \text { for } j \neq k, \\
& \text { and such that } Q_{j, 2} \cap F=\emptyset, \\
& m(\Omega) \leq \frac{C}{\alpha} \int_{\mathbb{R}^{n}}|f(x)|_{X} d x \text {, and } \\
& \frac{1}{\left|Q_{j}\right|} \int_{Q_{j}}|f(x)|_{X} d x \leq C \alpha .
\end{aligned}
$$

Here $Q_{j, 2}$ denotes, similarly as before, the double cube which has the same center as $Q_{j}$ but whose sides are twice as long as those of $Q$. We set

$$
g(x):= \begin{cases}f(x) & \text { for } x \in F, \\ \frac{1}{\left|Q_{j}\right|} \int_{Q_{j}} f(x) d x & \text { for } x \in Q_{j},\end{cases}
$$

and we set $b(x)=f(x)-g(x)$. Then

$$
\begin{aligned}
& b(x)=0 \text { for } x \in F, \text { and } \\
& \int_{Q_{j}} b(x) d x=0 \quad \text { for each cube } Q_{j} .
\end{aligned}
$$

Moreover, $g \in L^{1} \cap L^{\infty}(X),\|g\|_{L^{1}(X)} \leq\|f\|_{L^{1}(X)}$ and $\|g\|_{L^{\infty}(X)} \leq C \alpha$.

Since $T f=T g+T b$, it follows that

$$
\begin{aligned}
m\left(\left\{x \in \mathbb{R}^{n}:|T f(x)|_{Y}>\alpha\right\}\right) & \leq \\
& \leq m\left(\left\{x \in \mathbb{R}^{n}:|T g(x)|_{Y}>\frac{\alpha}{2}\right\}\right)+m\left(\left\{x \in \mathbb{R}^{n}:|\operatorname{Tb}(x)|_{Y}>\frac{\alpha}{2}\right\}\right),
\end{aligned}
$$

and it suffices to estimate both terms on the right hand side separately.

First, we estimate $T g$. First of all, $g \in L^{p}(X)$ and

$$
\|g\|_{L^{p}(X)}^{p}=\int_{\mathbb{R}^{n}}|g(x)|_{X}^{p} d x \leq C^{p-1} \alpha^{p-1}\|g\|_{L^{1}(X)} \leq C^{p-1} \alpha^{p-1}\|f\|_{L^{1}(X)}
$$

By using in addition the assumption of boundedness of $T$,

$$
\|T g\|_{L^{p}(Y)}^{p} \leq C^{p}\|g\|_{L^{p}(X)}^{p} \leq C \alpha^{p-1}\|f\|_{L^{1}(X)},
$$

and this implies

$$
m\left(\left\{x \in \mathbb{R}^{n}:|T g(x)|_{Y}>\frac{\alpha}{2}\right\}\right) \leq \frac{C}{\alpha}\|f\|_{L^{1}(X)} .
$$


Second, we estimate $T b$. Let $b_{j}=b \chi_{Q_{j}}$. Then $b=\sum_{j} b_{j}$ and it suffices to estimate $T b_{j}$.

Fix $x \in F$ and fix $j$. Since $\int_{Q_{j}} b=0$, we have

$$
T b_{j}(x)=\int_{Q_{j}}\left(K(x, y)-K\left(x, x_{j}\right)\right) b(y) d y,
$$

where $x_{j}$ is the center of the cube $Q_{j}$. In particular,

$$
\begin{aligned}
\int_{F}\left|T b_{j}(x)\right|_{Y} d x & \leq \int_{F} \int_{Q_{j}}\left|K(x, y)-K\left(x, x_{j}\right)\right|_{\mathcal{L}(X, Y)}|b(y)|_{X} d y d x \\
& =\int_{Q_{j}} \int_{F}\left|K(x, y)-K\left(x, x_{j}\right)\right|_{\mathcal{L}(X, Y)} d x|b(y)|_{X} d y \\
& \leq \int_{Q_{j}} \int_{Q_{j, 2}^{c}}\left|K(x, y)-K\left(x, x_{j}\right)\right|_{\mathcal{L}(X, Y)} d x|b(y)|_{X} d y \\
& \leq C_{K} \int_{Q_{j}}|b(y)|_{X} d y,
\end{aligned}
$$

where we have used the fact that $F$ is a subset of the complement of the double cube $Q_{j, 2}$. Of course, we also used that $K$ satisfies the first Hörmander integral condition (4.6). From the preceding estimate we obtain

$$
\begin{aligned}
\int_{F}|T b(x)|_{Y} d x & \leq \sum_{j} \int_{F}\left|T b_{j}(x)\right|_{Y} d x \\
& \leq C_{K} \int_{\Omega}|b(y)|_{X} d y \\
& \leq 2 C_{K}\|f\|_{L^{1}(X)} .
\end{aligned}
$$

This estimate implies

$$
m\left(\left\{x \in F:|T b(x)|_{Y}>\frac{\alpha}{2}\right\}\right) \leq \frac{C}{\alpha}\|f\|_{L^{1}(X)} .
$$

On the other hand,

$$
m\left(\left\{x \in \Omega:|T b(x)|_{Y}>\frac{\alpha}{2}\right\}\right) \leq m(\Omega) \leq \frac{C}{\alpha}\|f\|_{L^{1}(X)} .
$$

The preceding two estimates together give the estimate for $\mathrm{Tb}$.

The following proposition is a consequence of the proof of [9, Theorem 2]; see [29, Proof of Theorem 4.1]. 
Proposition 5.2. Let $T$ be a singular integral operator from $L^{p}(X)$ into $L^{p}(Y)$. Assume that $T$ satisfies the second Hörmander integral condition (4.7). Then there exists a constant $C \geq 0$ such that

$$
\|T f\|_{B M O(Y)} \leq C\|f\|_{L^{\infty}(X)} \quad \text { for every } f \in L^{p} \cap L^{\infty}(X) .
$$

Besides the two preceding weak estimates for the operator $T$, we also need the following version of Boyd's interpolation theorem from [10, Theorem 5.16, p. 153] for quasi-linear operators acting between Banach space valued $L^{p}$ spaces. It is a straightforward consequence of [10, Theorem 5.16, p.153] which is Boyd's interpolation theorem for quasi-linear operators acting on scalar $L^{p}$ spaces, if one uses a simple trick contained in the proof of [9, Lemma 1]; for details, see [22, Remark 3.12]. However, for the convenience of the reader, here we apply the trick to the original proof, which is, this way, recalled and extended in a unified argument.

Theorem 5.3. Let $1 \leq p<q \leq \infty$. Let $T: L^{p, 1} \cap L^{q, 1}(X) \rightarrow \mathcal{M}(Y)$ be a quasilinear operator, that is, an operator satisfying the inequality

$$
|T(f+g)|_{Y} \leq C\left(|T f|_{Y}+|T g|_{Y}\right) \text { for every } f, g \in L^{p, 1} \cap L^{q, 1}(X) \text { and some } C \geq 0 \text {. }
$$

Assume that $T$ satisfies the weak type $(p, p)$ and weak type $(q, q)$ estimates

$$
\|T f\|_{L^{p, \infty}(Y)} \leq C_{p}\|f\|_{L^{p, 1}(X)} \text { and }\|T f\|_{L^{q, \infty}(Y)} \leq C_{q}\|f\|_{L^{q, 1}(X)}
$$

for every $f \in L^{p, 1} \cap L^{q, 1}(X)$ and some constants $C_{p}, C_{q} \geq 0$. Then, for every r.i.BFS $\mathbb{E}$ with Boyd indices $p<p_{\mathbb{E}} \leq q_{\mathbb{E}}<q$ there exists a constant $C_{\mathbb{E}} \geq 0$ such that

$$
\|T f\|_{\mathbb{E}(Y)} \leq C_{\mathbb{E}}\|f\|_{\mathbb{E}(X)} \text { for every } f \in \mathbb{E}(X),
$$

that is, $T$ extends to a bounded operator $\mathbb{E}(X) \rightarrow \mathbb{E}(Y)$.

Proof. Let $f \in L^{p} \cap L^{q}(X)$. For fixed $t>0$, we define the functions

$$
\begin{aligned}
& f_{1}(x):=\min \left\{|f(x)|_{X}, f^{*}(t)\right\} \frac{f(x)}{|f(x)|_{X}} \text { and } \\
& f_{0}(x):=f(x)-f_{1}(x)=\left[|f(x)|_{X}-f^{*}(t)\right]^{+} \frac{f(x)}{|f(x)|_{X}},
\end{aligned}
$$

where we interprete $\frac{f(x)}{|f(x)|_{X}}=0$ if $f(x)=0$. Then, for every $s>0$,

$$
\begin{aligned}
f_{1}^{*}(s) & =\min \left\{f^{*}(s), f^{*}(t)\right\} \text { and } \\
f_{0}^{*} & =\left[f^{*}(s)-f^{*}(t)\right]^{+},
\end{aligned}
$$

so that

$$
\begin{aligned}
& \left\|f_{1}\right\|_{L^{q, 1}(X)}=\int_{0}^{\infty} s^{\frac{1}{q}} f_{1}^{*}(s) \frac{d s}{s}=q t^{\frac{1}{q}} f^{*}(t)+\int_{t}^{\infty} s^{\frac{1}{q}} f^{*}(s) \frac{d s}{s} \text { and } \\
& \left\|f_{0}\right\|_{L^{p, 1}(X)}=\int_{0}^{\infty} s^{\frac{1}{p}} f_{0}^{*}(s) \frac{d s}{s}=\int_{0}^{t} s^{\frac{1}{p}} f^{*}(s) \frac{d s}{s}-p t^{\frac{1}{p}} f^{*}(t) .
\end{aligned}
$$


By assumption and a straightforward estimate (compare with [10, Proposition 1.7 , p. 41]), for every $t>0$,

$$
(T f)^{*}(t) \leq C\left(\left(T f_{0}\right)^{*}\left(\frac{t}{2}\right)+\left(T f_{1}\right)^{*}\left(\frac{t}{2}\right)\right)
$$

The weak type estimates for $T$ yield

$$
\begin{aligned}
& \left(T f_{0}\right)^{*}\left(\frac{t}{2}\right) \leq C_{p}\left(\frac{t}{2}\right)^{-\frac{1}{p}}\left\|f_{0}\right\|_{L^{p, 1}(X)} \text { and } \\
& \left(T f_{1}\right)^{*}\left(\frac{t}{2}\right) \leq C_{q}\left(\frac{t}{2}\right)^{-\frac{1}{q}}\left\|f_{1}\right\|_{L^{q, 1}(X)} .
\end{aligned}
$$

By combining the preceding two estimates, we obtain

$$
(T f)^{*}(t) \leq C^{\prime}\left(\frac{1}{p} t^{-\frac{1}{p}}\left\|f_{0}\right\|_{L^{p, 1}(X)}+\frac{1}{q} t^{-\frac{1}{q}}\left\|f_{1}\right\|_{L^{q, 1}(X)}\right)
$$

with $C^{\prime}=C \max \left\{p C_{p} 2^{\frac{1}{p}}, q C_{q} 2^{\frac{1}{q}}\right\}$. By inserting the expressions from (5.1) and noting that the non-integral terms cancel, we obtain

$$
(T f)^{*}(t) \leq C^{\prime}\left(t^{-\frac{1}{p}} \int_{0}^{t} s^{\frac{1}{p}} f^{*}(s) \frac{d s}{s}+t^{-\frac{1}{q}} \int_{t}^{\infty} s^{\frac{1}{q}} f^{*}(s) \frac{d s}{s}\right)=: C^{\prime}\left(S_{p q} f^{*}\right)(t),
$$

where $S_{p q}$ is the so-called Calderón operator $\mathcal{M}^{+}(0, \infty) \rightarrow \mathcal{M}^{+}(0, \infty)$ (compare with [10, p. 142]). Let $\mathbb{E}$ be a r.i.BFS with Boyd indices $p<p_{E} \leq q_{\mathbb{E}}<q$, and let $\overline{\mathbb{E}}$ be its Luxembourg representation on the measure space $([0, \infty[, d t)$. It follows from [10, Theorem 5.15, p. 150] that the Calderón operator $S_{p q}$ is bounded on $\overline{\mathbb{E}}$, that is, there exists a constant $C_{\mathbb{E}}^{\prime} \geq 0$ such that

$$
\left\|S_{p q} g\right\|_{\overline{\mathbb{E}}} \leq C_{\mathbb{E}}^{\prime}\|g\|_{\bar{E}} \text { for every } g \in \overline{\mathbb{E}} .
$$

By combining the preceding two estimates and by using the definition of the norm in $\mathbb{E}(X)$ and $\mathbb{E}(Y)$, we finally obtain for every $f \in L^{p, 1} \cap L^{q, 1}(X)$ the estimate

$$
\begin{aligned}
\|T f\|_{\mathbb{E}(Y)} & =\left\||T f(\cdot)|_{Y}\right\|_{\mathbb{E}} \\
& =\left\|(T f)^{*}\right\|_{\overline{\mathbb{E}}} \\
& \leq C^{\prime}\left\|S_{p q} f^{*}\right\|_{\overline{\mathbb{E}}} \\
& \leq C^{\prime} C_{\mathbb{E}}^{\prime}\left\|f^{*}\right\|_{\overline{\mathbb{E}}} \\
& =C^{\prime} C_{\mathbb{E}}^{\prime}\left\||f(\cdot)|_{X}\right\|_{\mathbb{E}} \\
& =C^{\prime} C_{\mathbb{E}}^{\prime}\|f\|_{\mathbb{E}(X),}
\end{aligned}
$$

and this was the claim.

Proof of Theorem 4.2. The assertion (i) follows from Proposition 5.1 and Theorem 5.3. For assertion (ii), one applies in addition Proposition 5.2 and [29, Proposition 3.4]. 


\section{Proof of Theorems 4.3 And 4.4}

In order to avoid nonnecessary technicalities we change, in this section, the notation of the norm in $\mathbb{R}^{n}$. In this section, $|\cdot|$ denotes the $\infty$-norm on $\mathbb{R}^{n}$, that is, $|x|=\sup \left|x_{i}\right|$ for $x=\left(x_{i}\right) \in \mathbb{R}^{n}$. In this way, balls (for this norm) are cubes with sides parallel to the coordinate axes.

Let $K$ be a standard kernel. For every $\varepsilon>0$ we define the truncated kernels $K_{\varepsilon}$ by

$$
K_{\varepsilon}(x, y)= \begin{cases}K(x, y) & \text { if }|x-y| \geq \varepsilon, \\ 0 & \text { else, }\end{cases}
$$

and we define the associated integral operators $T_{\varepsilon}$ by

$$
T_{\varepsilon} f(x)=\int_{\mathbb{R}^{n}} K_{\varepsilon}(x, y) f(y) d y .
$$

Note that, by the first standard condition and since $p<\infty$, the above integral converges absolutely for every $x \in \mathbb{R}^{n}$ and every $f \in L^{p}(X)$.

Next, we define the maximal function

$$
T^{*} f(x):=\sup _{Q}\left|\int_{Q^{c}} K(x, y) f(y) d y\right|_{Y^{\prime}},
$$

where the supremum is taken over all cubes $Q$ with center in $x$, and where $Q^{c}$ denotes the complement of $Q$. Note that

$$
T^{*} f(x)=\sup _{\varepsilon>0}\left|T_{\varepsilon} f(x)\right|_{Y}
$$

where $T_{\varepsilon}$ is defined as above.

Proposition 6.1. For every $r>0$ there exists a constant $C \geq 0$ such that

$$
T^{*} f \leq C\left(\left(M|T f(\cdot)|_{Y}^{r}\right)^{\frac{1}{r}}+M f\right) \text { pointwise everywhere in } \mathbb{R}^{n} \text {. }
$$

Proof. Fix $\bar{x} \in \mathbb{R}^{n}$. If $\left(M|T f(\cdot)|_{\gamma}^{r}\right)^{\frac{1}{r}}(\bar{x})=\infty$ or if $M f(\bar{x})=\infty$, then there remains nothing to prove. Hence, we can assume that $\left(M|T f(\cdot)|_{\gamma}^{r}\right)^{\frac{1}{r}}(\bar{x})<\infty$ and $M f(\bar{x})<$ $\infty$.

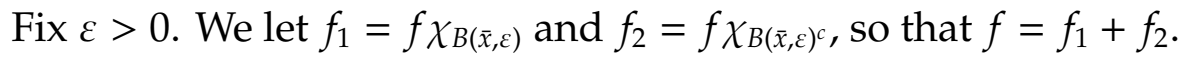

First of all, whenever $|x-\bar{x}| \leq \frac{\varepsilon}{2}$, then

$$
\begin{aligned}
\left|T f_{2}(\bar{x})-T f_{2}(x)\right|_{Y} & \leq \int_{B(\bar{x}, \varepsilon)^{c}}|K(\bar{x}, y)-K(x, y)|_{\mathcal{L}(X, Y)}|f(y)|_{X} d y \\
& \leq C_{K} \sum_{k=0}^{\infty} \int_{B\left(\bar{x}, 2^{k+1} \varepsilon\right) \backslash B\left(\bar{x}, 2^{k} \varepsilon\right)} \frac{|\bar{x}-x|^{\delta}}{|\bar{x}-y|^{n+\delta}}|f(y)|_{X} d y
\end{aligned}
$$


SINGULAR INTEGRAL OPERATORS AND EXTRAPOLATION OF MAXIMAL REGULARITY 19

$$
\begin{aligned}
& \leq C_{K} 2^{-\delta} \sum_{k=0}^{\infty} \frac{\varepsilon^{\delta}}{2^{k(n+\delta)} \varepsilon^{n+\delta}} \int_{B\left(\bar{x}, 2^{k+1} \varepsilon\right) \backslash B\left(\bar{x}, 2^{k} \varepsilon\right)}|f(y)|_{X} d y \\
& \leq C_{K} 2^{n-\delta} \sum_{k=0}^{\infty} 2^{-k \delta} \frac{1}{\left(2^{k+1} \varepsilon\right)^{n}} \int_{B\left(\bar{x}, 2^{k+1} \varepsilon\right)}|f(y)|_{X} d y \\
& \leq C M f(\bar{x}) .
\end{aligned}
$$

Hence, whenever $|x-\bar{x}| \leq \frac{\varepsilon}{2}$, then

$$
\begin{aligned}
\left|T_{\varepsilon} f(\bar{x})\right|_{Y} & =\left|T f_{2}(\bar{x})\right|_{Y} \\
& \leq\left|T f_{2}(\bar{x})-T f_{2}(x)\right|_{Y}+|T f(x)|_{Y}+\left|T f_{1}(x)\right|_{Y} \\
& \leq C M f(\bar{x})+|T f(x)|_{Y}+\left|T f_{1}(x)\right|_{Y} .
\end{aligned}
$$

Now, for every $r>0$ and every $\alpha>0$,

$$
\begin{aligned}
m\left(\left\{x \in B\left(\bar{x}, \frac{\varepsilon}{2}\right):|T f(x)|_{Y}>\alpha\right\}\right) & \leq \alpha^{-r} \int_{B\left(\bar{x}, \frac{\varepsilon}{2}\right)}|T f(x)|_{Y}^{r} d x \\
& \leq \alpha^{-r} m\left(B\left(\bar{x}, \frac{\varepsilon}{2}\right)\right) M|T f(\cdot)|_{Y}^{r}(\bar{x}) .
\end{aligned}
$$

Thus, if $\alpha \geq 4^{\frac{1}{r}}\left(M|T f(\cdot)|^{r}(\bar{x})\right)^{\frac{1}{r}}$, then

$$
m\left(\left\{x \in B\left(\bar{x}, \frac{\varepsilon}{2}\right):|T f(x)|_{Y}>\alpha\right\}\right) \leq \frac{1}{4} m\left(B\left(\bar{x}, \frac{\varepsilon}{2}\right)\right) .
$$

One also has

$$
\begin{aligned}
m\left(\left\{x \in B\left(\bar{x}, \frac{\varepsilon}{2}\right):\left|T f_{1}(x)\right|_{Y}>\alpha\right\}\right) & \leq \frac{C}{\alpha} \int_{B\left(\bar{x}, \frac{\varepsilon}{2}\right)}|f(y)|_{X} d y \\
& \leq \frac{C}{\alpha} m\left(B\left(\bar{x}, \frac{\varepsilon}{2}\right)\right) M f(\bar{x}) .
\end{aligned}
$$

Thus, if $\alpha \geq 4 C M f(\bar{x})$, then

$$
m\left(\left\{x \in B\left(\bar{x}, \frac{\varepsilon}{2}\right):\left|T f_{1}(x)\right|_{Y}>\alpha\right\}\right) \leq \frac{1}{4} m\left(B\left(\bar{x}, \frac{\varepsilon}{2}\right)\right) .
$$

Therefore, if

$$
\alpha=\max \left\{4^{\frac{1}{r}}\left(M|T f(\cdot)|_{Y}^{r}(\bar{x})\right)^{\frac{1}{r}}, 4 C M f(\bar{x})\right\},
$$

then there exists $x \in B\left(\bar{x}, \frac{\varepsilon}{2}\right)$ such that

$$
|T f(x)|_{Y} \leq \alpha \text { and }\left|T f_{1}(x)\right|_{Y} \leq \alpha .
$$

Inserting this into (6.3) yields the claim.

From the preceding proposition we obtain the following statement. 
Corollary 6.2. The maximal operator $T^{*}$ is weak $(1,1)$ in the sense that there exists a constant $C \geq 0$ such that for every $\alpha>0$ one has

$$
m\left(\left\{x \in \mathbb{R}^{n}: T^{*} f(x)>\alpha\right\}\right) \leq \frac{C}{\alpha} \int_{\mathbb{R}^{n}}|f(x)|_{X} d x .
$$

Proof. By Proposition 6.1 and since the Hardy-Littlewood maximal operator is weak $(1,1)$, for every $\alpha>0$

$$
\begin{aligned}
& m\left(\left\{x \in \mathbb{R}^{n}: T^{*} f>\alpha\right\}\right) \leq \\
& \leq m\left(\left\{x \in \mathbb{R}^{n}:\left(M|T f(\cdot)|_{Y}^{r}\right)^{\frac{1}{r}}(x)>\frac{\alpha}{2 C}\right\}\right)+m\left(\left\{x \in \mathbb{R}^{n}: M f(x)>\frac{\alpha}{2 C}\right\}\right. \\
& \leq m\left(\left\{x \in \mathbb{R}^{n}:\left(M|T f(\cdot)|_{Y}^{r}\right)^{\frac{1}{r}}(x)>\frac{\alpha}{2 C}\right\}\right)+\frac{C}{\alpha}\|f\|_{L^{1}},
\end{aligned}
$$

where $C \geq 0$ is the constant from (6.1). It suffices to estimate the first term on the right-hand side of this inequality. Recall that a function $F \in \mathcal{M}$ satisfies the the weak $(1,1)$ inequality

$$
m\left(\left\{x \in \mathbb{R}^{n}:|F(x)| \geq \alpha\right\}\right) \leq \frac{A}{\alpha} \quad \text { for every } \alpha>0
$$

if and only if for some / all $r \in(0,1)$ the function $|F|^{r}$ belongs to the Lorentz space $L^{\frac{1}{r}, \infty}$ and $\left\||F|^{r}\right\|_{L^{\frac{1}{r}, \infty}} \leq C A^{r}$ for some constant $C$ which depends only on r; see [47, p. 36] or Example 2.6 together with [10, Lemma 4.5, p.219]. Since the operator $T$ is weak $(1,1)$ by Proposition 5.1 , the above equivalence (applied with $F=|T f|)$ implies that $|T f|^{r}$ belongs to $L^{\frac{1}{r}, \infty}$ and has norm $\leq C\|f\|_{L^{1}}^{r}$. Now, by the Marcinkiewicz interpolation theorem [10, Theorem 4.13, p.225], the Hardy-Littlewood maximal operator is bounded on $L^{\frac{1}{r}, \infty}$ if $0<r<1$. Using this and again the above equivalence (now applied with $F=\left(M|T f(\cdot)|_{\gamma}^{r}\right)^{\frac{1}{r}}$ ), one obtains the desired estimate

$$
m\left(\left\{x \in \mathbb{R}^{n}:\left(M|T f(\cdot)|_{Y}^{r}\right)^{\frac{1}{r}}(x) \geq \frac{\alpha}{2 C}\right\}\right) \leq \frac{C}{\alpha}\|f\|_{L^{1}} .
$$

Proof of Theorem 4.4. In this proof, we use the maximal function given by

$$
\bar{T}^{*} f(x):=\max \left\{T^{*} f(x),|T f(x)|_{Y}\right\} .
$$

Fix a weight $w \in A_{\infty}$. We shall first prove that there exist $C \geq 0$ and $\delta>0$ such that for every $\alpha>0$, and every $\gamma>0$ small enough the inequality

$$
w\left(\left\{x \in \mathbb{R}^{n}: \bar{T}^{*} f(x)>2 \alpha \text { and } M f(x) \leq \gamma \alpha\right\}\right) \leq C \gamma^{\delta} w\left(\left\{x \in \mathbb{R}^{n}: \bar{T}^{*} f(x)>\alpha\right\}\right)
$$

holds. 
First, we can assume that $w\left(\left\{x \in \mathbb{R}^{n}: \bar{T}^{*} f(x)>\alpha\right\}\right) \neq 0$, for otherwise the above inequality is clearly satisfied. Since the measure $w(x) d x$ is outer regular, there exists an open set $U_{\alpha}$ such that

$$
\begin{aligned}
& \left\{x \in \mathbb{R}^{n}: \bar{T}^{*} f(x)>\alpha\right\} \subseteq U_{\alpha} \text { and } \\
& w\left(U_{\alpha}\right) \leq 2 w\left(\left\{x \in \mathbb{R}^{n}: \bar{T}^{*} f(x)>\alpha\right\}\right) .
\end{aligned}
$$

By Whitney's lemma [46, Theorem 3, p. 16] (see in addition [46, Chapter VI, p. $168])$, there exists a sequence $\left(Q_{k}\right)$ of mutually disjoint cubes with sides parallel to the coordinate axes such that $U_{\alpha}=\bigcup_{k} \bar{Q}_{k}$ and such that $Q_{k, 4}$ (the cube which has the same center as the cube $Q_{k}$ and satisfies diam $Q_{k, 4}=4 \operatorname{diam} Q_{k}$ ) has nonempty intersection with $U_{\alpha}^{c}$. The cubes $Q_{k}$ are of the form $B\left(\bar{x}_{k}, r_{k}\right)$ for some center $\bar{x}_{k}$ and some radius $r_{k}>0$, and hence $Q_{k, 4}=B\left(\bar{x}_{k}, 4 r_{k}\right)$.

We prove that there exists a constant $C \geq 0$ which is independent of $f$ such that for every $\gamma>0$ small enough and every $k$,

$$
m\left(\left\{x \in Q_{k}: \bar{T}^{*} f(x)>2 \alpha \text { and } M f(x) \leq \gamma \alpha\right\}\right) \leq C \gamma m\left(Q_{k}\right) .
$$

We may in fact assume that $\gamma$ is small, since the above inequality is trivial for $\gamma \geq C^{-1}$.

Fix $k$. We may assume that there exists $\xi_{k} \in Q_{k}$ such that $M f\left(\xi_{k}\right) \leq \gamma \alpha$, because otherwise the inequality (6.6) is obviously satisfied. Moreover, since $Q_{k, 4} \cap U_{\alpha}^{c}$ is nonempty, there exists $x_{k} \in Q_{k, 4}$ such that

$$
\bar{T}^{*} f\left(x_{k}\right) \leq \alpha .
$$

Now let $\tilde{Q}_{k}:=B\left(x_{k}, 16 r_{k}\right)$ be the cube centered at $x_{k}$ and satisfying diam $\tilde{Q}_{k}=$ 16 diam $Q_{k}$. Define $f_{1}=f \chi_{\tilde{Q}_{k}}$ and $f_{2}=f \chi_{\tilde{Q}_{k}^{c}}$, so that $f=f_{1}+f_{2}$. By subadditivity of the maximal operator $\bar{T}^{*}$, we have

$$
\begin{aligned}
& m\left(\left\{x \in Q_{k}: \bar{T}^{*} f(x)>2 \alpha \text { and } M f(x) \leq \gamma \alpha\right\}\right) \leq \\
& \leq m\left(\left\{x \in Q_{k}: \bar{T}^{*} f_{1}(x)>\frac{\alpha}{2} \text { and } M f(x) \leq \gamma \alpha\right\}\right)+ \\
& \quad+m\left(\left\{x \in Q_{k}: \bar{T}^{*} f_{2}(x)>\frac{3 \alpha}{2} \text { and } M f(x) \leq \gamma \alpha\right\}\right),
\end{aligned}
$$

and it suffices to estimate the two terms on the right-hand side of this inequality.

Since $\xi_{k} \in Q_{k} \subseteq \tilde{Q}_{k}$, it follows that

$$
\frac{1}{m\left(Q_{k}\right)} \int_{\mathbb{R}^{n}}\left|f_{1}(y)\right|_{X} d y=\frac{1}{m\left(Q_{k}\right)} \int_{\tilde{Q}_{k}}|f(y)|_{X} d y \leq 16^{n} M f\left(\xi_{k}\right) \leq 16^{n} \gamma \alpha,
$$

so that the weak $(1,1)$ estimates from Proposition 5.1 and Corollary 6.2 yield

$$
m\left(\left\{x \in \mathbb{R}^{n}: \bar{T}^{*} f_{1}>\frac{\alpha}{2}\right\}\right) \leq \frac{2 C}{\alpha} \int_{\mathbb{R}^{n}}\left|f_{1}(y)\right|_{X} d y \leq C \gamma m\left(Q_{k}\right)
$$


for some constant $C \geq 0$ which is independent of $f, \gamma, k$ and $\alpha$. Next, we shall estimate, for small $\gamma>0$, the second term on the right-hand side of (6.7). Fix $x \in Q_{k}=B\left(\bar{x}_{k}, r_{k}\right)$ and $\varepsilon>0$. Then

$$
\begin{aligned}
\left|T_{\varepsilon} f_{2}(x)\right|_{Y}= & \left|\int_{B(x, \varepsilon)^{c}} K(x, y) f_{2}(y) d y\right|_{Y} \\
\leq & \left|\int_{B\left(x_{k}, \varepsilon\right)^{c}} K\left(x_{k}, y\right) f_{2}(y) d y\right|_{Y} \\
& +\int_{B\left(x_{k}, \varepsilon\right)^{c}}\left|K\left(x_{k}, y\right)-K(x, y)\right|_{\mathcal{L}(X, Y)}\left|f_{2}(y)\right|_{X} d y \\
& +\int_{B(x, \varepsilon) \Delta B\left(x_{k}, \varepsilon\right)}|K(x, y)|_{\mathcal{L}(X, Y)}\left|f_{2}(y)\right|_{X} d y \\
= & : I_{1}+I_{2}+I_{3},
\end{aligned}
$$

where $B(x, \varepsilon) \Delta B\left(x_{k}, \varepsilon\right)$ denotes the symmetric difference of $B(x, \varepsilon)$ and $B\left(x_{k}, \varepsilon\right)$. We have

$$
I_{1}=\left|\int_{\left(B\left(x_{k}, \varepsilon\right) \cup B\left(x_{k}, 8 r_{k}\right)\right)^{c}} K\left(x_{k}, y\right) f(y) d y\right|_{Y} \leq \bar{T}^{*} f\left(x_{k}\right) \leq \alpha
$$

by the choice of $x_{k}$, since $B\left(x_{k}, \varepsilon\right) \cup B\left(x_{k}, 8 r_{k}\right)=B\left(x_{k}, \sup \left\{\varepsilon, 8 r_{k}\right\}\right)$ is a cube centered at $x_{k}$, and by the definition of $\bar{T}^{*}$. Furthermore, by using $\xi_{k} \in Q_{k} \subseteq \tilde{Q}_{k}$ again, and by proceeding similarly as in the estimates (6.2), one obtains

$$
I_{2} \leq C M f\left(\xi_{k}\right) \leq C \gamma \alpha .
$$

Note that $I_{3}=0$ whenever $\varepsilon \leq 16 r_{k}$. On the other hand, for $\varepsilon \geq 16 r_{k}$ one has $B(x, \varepsilon) \triangle B\left(x_{k}, \varepsilon\right) \subseteq B(x, 2 \varepsilon) \backslash B(x, \varepsilon / 2)$, and therefore,

$$
\begin{aligned}
I_{3} & \leq \int_{B(x, 2 \varepsilon) \backslash B(x, \varepsilon / 2)} \frac{C_{K}}{|x-y|^{n}}|f(y)|_{X} d y \\
& \leq \frac{C_{K} 2^{n}}{\varepsilon^{n}} \int_{B(x, 2 \varepsilon)}|f(y)|_{X} d y \\
& \leq C M f\left(\xi_{k}\right) \leq C \gamma \alpha .
\end{aligned}
$$

We note that the preceding estimates can also be made for $\varepsilon=0$ if one interpretes $T_{0}=T$. Taking all the above estimates together, and taking the supremum over $\varepsilon \geq 0$, we find that there exists a constant $C \geq 0$ which is independent of $f, \alpha, k, \gamma$ such that for every $x \in Q_{k}$,

$$
\left|\bar{T}^{*} f_{2}(x)\right|_{Y} \leq C \gamma \alpha+\alpha .
$$

Taking $\gamma>0$ so small that $C \gamma<\frac{1}{2}$, it follows that

$$
m\left(\left\{x \in Q_{k}: \bar{T}^{*} f_{2}(x)>\frac{3 \alpha}{2} \text { and } M f(x) \leq \gamma \alpha\right\}\right)=0 .
$$


As a consequence, we have proved (6.6). Now, since the weight $w$ belongs to $A_{\infty}$, there exist constants $\delta>0$ such that

$$
\frac{w(E)}{w(Q)} \leq C\left(\frac{m(E)}{m(Q)}\right)^{\delta} \text { for every cube } Q \text { and every measurable } E \subseteq Q .
$$

Hence, the estimate (6.6) implies that, for every $k$,

$$
w\left(\left\{x \in Q_{k}: \bar{T}^{*} f(x)>2 \alpha \text { and } M f(x) \leq \gamma \alpha\right\}\right) \leq C \gamma^{\delta} w\left(Q_{k}\right) .
$$

Summing up in $k$ and recalling the inequality (6.5) yields the estimate (6.4).

Now the rest of the proof is standard. By (6.4), for every $f \in L^{1} \cap L^{p}(X)$,

$$
\begin{aligned}
& \int_{\mathbb{R}^{n}} \bar{T}^{*} f(x)^{p} w(x) d x= \\
& =C \int_{0}^{\infty} \alpha^{p-1} w\left(\left\{\bar{T}^{*} f>2 \alpha\right\}\right) d \alpha \\
& \leq C \int_{0}^{\infty} \alpha^{p-1} w(\{M f>\gamma \alpha\}) d \alpha+C \gamma^{\delta} \int_{0}^{\infty} \alpha^{p-1} w\left(\left\{\bar{T}^{*} f>\alpha\right\}\right) d \alpha \\
& =C \int_{\mathbb{R}^{n}} M f(x)^{p} w(x) d x+C \gamma^{\delta} \int_{\mathbb{R}^{n}} \bar{T}^{*} f(x)^{p} w(x) d x .
\end{aligned}
$$

Taking $\gamma>0$ so small that $C \gamma^{\delta} \leq \frac{1}{2}$, we obtain the claim.

We are now in the position to prove Theorem 4.3.

Proof of Theorem 4.3. The statement (i) is a direct consequence of Theorem 4.4 and Theorem 4.5 applied to the family $\mathcal{F}=\left\{\left(|T f|_{Y},|M f|_{X}\right): f \in L^{p} \cap L_{w}^{p}(X)\right\}$.

The statement (ii) follows from the observation

$$
M f=M|f|_{X} \quad \text { and }\|f\|_{\mathbb{E}_{w}(X)}=\left\||f|_{X}\right\|_{\mathbb{E}_{w}}
$$

(see also Section 3) and the corresponding statement for scalar-valued functions which is [17, Theorem 2.3 (i)].

The statement (iii) follows immediately from (i) and (ii).

\section{Maximal Regularity of abstract Cauchy problems}

Singular integral operators with operator valued kernels arise naturally in the context of evolution equations when solutions are represented by convolutions with semigroups, evolution families, sine families or other solution families. We apply the abstract extrapolation theorem (Theorem 4.3) in a situation in which we can show that the kernel is a standard kernel. 
7.1. The nonautonomous, first order Cauchy problem on the line. We consider the non-autonomous, first order Cauchy problem

$$
\dot{u}+A(t) u=f \quad \text { on } \mathbb{R},
$$

where the operators $A(t)$ are closed, linear, densely defined operators on a Banach space $X$. Given a r.i.BFS $\mathbb{E}=\mathbb{E}(\mathbb{R})$ and a weight $w \in A_{p_{\mathbb{E}}}$, we say that this problem has $\mathbb{E}_{w}$-maximal regularity, if for every $f \in \mathbb{E}_{w}(X)=\mathbb{E}_{w}(\mathbb{R} ; X)$ there exists a unique, absolutely continuous solution $u: \mathbb{R} \rightarrow X$ for which $\dot{u}$ and $A(\cdot) u$ belong to $\mathbb{E}_{w}(X)$. The term "maximal regularity" refers here to the property that the two terms on the left-hand side of (7.1) have the same regularity as the given right-hand side. If the problem has $\mathbb{E}_{w}$-maximal regularity, then, by the Closed Graph Theorem, there exists a constant $C \geq 0$ such that

$$
\|u(0)\|_{X}+\|\dot{u}\|_{\mathbb{E}_{w}(X)}+\|A(\cdot) u\|_{\mathbb{E}_{w}(X)} \leq C\|f\|_{\mathbb{E}_{w}(X)} .
$$

We point out that here exist a few results in the literature about $L^{p}$-maximal regularity of nonautonomous Cauchy problems on the interval. If the domains of the operators $A(t)$ do not depend on time, we mention [42], [3], [5], and in the case of time-depending domains, we refer to [30], [31], [40], [6] and [27].

Here we consider the case in which the domains may depend on time. We assume that the family $(A(t))_{t \in \mathbb{R}}$ satisfies the so-called Kato-Tanabe conditions, that is

I. the $A(t)$ are uniformly sectorial in the sense that there exist constants $\theta \in$ $\left(0, \frac{\pi}{2}\right)$ and $C \geq 0$ such that for every $t \in \mathbb{R}_{+}$

$$
\sigma(A(t)) \subseteq \Sigma_{\theta}:=\{z \in \mathbb{C}:|\arg z|<\theta\}
$$

and for every $t \in \mathbb{R}_{+}, z \notin \Sigma_{\theta}$

$$
|R(z, A(t))|_{\mathcal{L}(X)} \leq \frac{C}{1+|z|},
$$

II. the function $R(z, A(\cdot))$ is differentiable and there exist constants $\varrho \in[0,1)$, $C \geq 0$ such that for every $t \in \mathbb{R}_{+}, z \notin \Sigma_{\theta}$

$$
\left|\frac{d}{d t} R(z, A(t))\right|_{\mathcal{L}(X)} \leq \frac{C}{1+|z|^{1-\varrho}} .
$$

We assume in addition that

III. there exists $\alpha \in(0,1]$ such that $A(\cdot)^{-1} \in C^{1, \alpha}\left(\mathbb{R}_{+} ; \mathcal{L}(X)\right)$, that is, the derivative $\frac{d}{d t} A(\cdot)^{-1}$ is uniformly Hölder continuous of order $\alpha$.

By [34], [49, Section 5.3], if the family $(A(t))$ satisfies the Kato-Tanabe conditions I. and II., then there exists a family $(U(t, s))_{t \geq s} \subseteq \mathcal{L}(X)$ such that

(i) for every $r \leq s \leq t$ one has $U(t, t)=I$ and $U(t, r)=U(t, s) U(s, r)$,

(ii) for every $x \in X$ the function $U(\cdot, \cdot) x$ is continuous, 
(iii) there exist $C \geq 0, \omega \in \mathbb{R}$ such that for every $t \geq s$ one has

$$
|U(t, s)|_{\mathcal{L}(X)} \leq C e^{\omega(t-s)},
$$

(iv) for every $x \in X$ and every $t>s$ the partial derivative $\frac{\partial}{\partial t} U(t, s) x$ exists, $U(t, s) x \in D(A(t))$ and $\frac{\partial}{\partial t} U(t, s)+A(t) U(t, s)=0$. Moreover, there exists $C \geq 0$ such that for every $t>s$ with $t-s \leq 1$

$$
|A(t) U(t, s)|_{\mathcal{L}(X)}=\left|\frac{\partial}{\partial t} U(t, s)\right|_{\mathcal{L}(X)} \leq \frac{C}{t-s} .
$$

In addition, by [2, Theorem 1.6],

(v) for every $t>s$ and every $x \in D(A(s))$ the partial derivative $\frac{\partial}{\partial s} U(t, s) x$ exists and $\frac{\partial}{\partial s} U(t, s)-U(t, s) A(s)=0$. Moreover, the operator $U(t, s) A(s)$ extends to a bounded linear operator on $X$, and there exists $C \geq 0$ such that for every $t>s$ with $t-s \leq 1$

$$
|U(t, s) A(s)|_{\mathcal{L}(X)}=\left|\frac{\partial}{\partial s} U(t, s)\right|_{\mathcal{L}(X)} \leq \frac{C}{t-s} .
$$

Finally, by [48, Theorem 1],

(vi) the function $t \mapsto A(t) U(t, s)$ is locally Hölder continuous for $t>s$. More precisely, for every $\varepsilon>0$ there exists a constant $C_{\varepsilon} \geq 0$ such that for every $t \geq t^{\prime}>s$ with $t-s \leq 1$

$$
\begin{aligned}
\mid A & (t) U(t, s)-\left.A\left(t^{\prime}\right) U\left(t^{\prime}, s\right)\right|_{\mathcal{L}(X)} \leq \\
\leq & C_{\varepsilon}\left\{\frac{t-t^{\prime}}{(t-s)\left(t^{\prime}-s\right)^{\varrho}}+\frac{t-t^{\prime}}{t-s}+\frac{\left(t-t^{\prime}\right)^{1-\varrho}}{(t-s)^{\varrho}}+\frac{\left(t-t^{\prime}\right)^{\alpha}}{(t-s)^{\varrho}}\left(1+\log \frac{t-s}{t-t^{\prime}}\right)+\right. \\
& +\frac{t-t^{\prime}}{(t-s)^{1+\varrho}}+\frac{t-t^{\prime}}{(t-s)\left(t^{\prime}-s\right)}+\frac{t-t^{\prime}}{(t-s)^{\varrho}\left(t^{\prime}-s\right)}+ \\
& +\frac{\left(t-t^{\prime}\right)^{1-\varrho}}{\left(t^{\prime}-s\right)^{\varrho-\alpha}}+\frac{\left(t-t^{\prime}\right)^{1-\varepsilon}}{\left(t^{\prime}-s\right)^{1+\varrho-\varepsilon}}+\frac{\left(t-t^{\prime}\right)^{1-\varrho-\varepsilon}}{\left(t^{\prime}-s\right)^{\varrho-\varepsilon}}+ \\
& +\frac{t-t^{\prime}}{(t-s)\left(t^{\prime}-s\right)^{\varrho}} \log \frac{t-s}{t-t^{\prime}}+\frac{t-t^{\prime}}{t-s} \log \frac{t-s}{t-t^{\prime}}+ \\
& \left.+\frac{\left(t-t^{\prime}\right)^{1-\varrho}}{(t-s)^{\varrho}} \log \frac{t-s}{t-t^{\prime}}+\frac{\left(t-t^{\prime}\right)^{\alpha}}{(t-s)^{\varrho}}\left(\log \frac{t-s}{t-t^{\prime}}\right)^{2}\right\} .
\end{aligned}
$$

In fact, [48, Theorem 1] only states the Hölder continuity, but the additional precise estimates are given in its proof; see [48, Step 1 of the proof of Theorem 1, p. 549]. Also, all the above estimate are only given for operator families defined on finite intervals. However, the constants appearing in the estimates depend only on the constants in conditions I.-III. (which are uniform) and on the length of the interval; this is reflected in the restriction $t-s \leq 1$ in the 
estimates in (iv)-(vi).

We call a family $(U(t, s))_{t \geq s}$ satisfying the properties (i) and (ii) an evolution family. By property (iv), for every $s \in \mathbb{R}$ and every $x \in X$ the unique solution of the initial-value problem

$$
\dot{u}+A(t) u=0 \quad \text { on }[s, \infty), \quad u(s)=x,
$$

is given by $u(t)=U(t, s) x$. This is a motivation to say that the evolution family $(U(t, s))_{t \geq s}$ is generated by $(A(t))$.

Corollary 7.1. Assume the conditions I.-III. and assume that the evolution family generated by $(A(t))$ is uniformly exponentially stable in the sense that there exist $C \geq 0$ and $\omega>0$ such that

$$
|U(t, s)|_{\mathcal{L}(X)} \leq C e^{-\omega(t-s)} \text { for every } t \geq s .
$$

If the problem (7.1) has $L^{p}$-maximal regularity for some $p \in(1, \infty)$, then it has $\mathbb{E}_{w}$-maximal regularity for every rearrangement invariant Banach space $\mathbb{E}$ satisfying $1<p_{\mathbb{E}} \leq q_{\mathbb{E}}<\infty$ and every weight $w \in A_{p_{\mathbb{E}}}$.

Proof. First, since $(U(t, s))_{t \geq s}$ is uniformly exponentially stable, for every $f \in$ $L^{p}(X)$ the unique solution $u$ of the inhomogeneous problem (7.1) is given by the variation of constants formula

$$
u(t)=\int_{-\infty}^{t} U(t, s) f(s) d s, \quad t \in \mathbb{R} ;
$$

compare with [41, Theorem 7.1, p.168]. Second, by the assumption of $L^{p_{-}}$ maximal regularity and by the closed graph theorem, the operator $T: L^{p}(X) \rightarrow$ $L^{p}(X), f \mapsto A(\cdot) u$ is well defined and bounded. In fact, this operator is bounded if and only if the problem (7.1) has $L^{p}$-maximal regularity, and similarly, this operator extends to a bounded operator on $\mathbb{E}_{w}(X)$ if and only if the problem (7.1) has $\mathbb{E}_{w}$-maximal regularity. We therefore have to show that $T$ extends (extrapolates) to a bounded operator on $\mathbb{E}_{w}$.

We set

$$
K(t, s)= \begin{cases}A(t) U(t, s) & \text { for } t>s, \\ 0 & \text { else. }\end{cases}
$$

Then $K \in L_{\text {loc }}^{1}\left(\mathbb{R}^{2} \backslash \Delta ; \mathcal{L}(X)\right)$, and the representation formula for the solutions $u$ yields

$$
T f(t)=\int_{\mathbb{R}} K(t, s) f(s) d s,
$$

at least for every $t \in \mathbb{R}$ and every $f \in L^{p}(X)$ with compact support outside $t$. Hence, $T$ is a singular integral operator. The claim follows from Theorem 4.3 once we can prove that $K$ is a standard kernel. 
Let $t, s \in \mathbb{R}$. By property (iv),

$$
|K(t, s)|_{\mathcal{L}(X)} \leq C \frac{1}{t-s} \quad \text { if } 0<t-s \leq 1 .
$$

By property (i), (iv) and the uniform exponential stability,

$$
|K(t, s)|_{\mathcal{L}(X)}=|K(t, t-1) U(t-1, s)|_{\mathcal{L}(X)} \leq C^{2} e^{\omega} e^{-\omega(t-s)} \quad \text { if } t-s \geq 1 .
$$

Finally,

$$
|K(t, s)|_{\mathcal{L}(X)}=0 \text { if } t-s<0 .
$$

Taking all three estimates together, we see that $K$ satisfies the first standard condition.

In order to prove that $K$ satisfies the second standard condition, observe first that there exists a constant $C \geq 0$ such that for every $t>s$

$$
\left|\frac{\partial}{\partial s} U(t, s)\right|_{\mathcal{L}(X)}=|U(t, s) A(s)|_{\mathcal{L}(X)} \leq \frac{C}{t-s},
$$

that is, the estimate from property $(\mathrm{v})$ holds without the restriction $t-s \leq 1$. In fact, if $t-s>1$, then

$$
|U(t, s) A(s)|_{\mathcal{L}(X)}=|U(t, s+1) U(s+1, s) A(s)|_{\mathcal{L}(X)} \leq C^{2} e^{\omega} e^{-\omega(t-s)}
$$

by property $(\mathrm{v})$ and the uniform exponential stability of $(U(t, s))_{t \geq s}$.

Let now $s, s^{\prime}, t \in \mathbb{R}$ be such that $\left|s-s^{\prime}\right| \leq \frac{1}{2}|t-s|$. Note that $\frac{1}{2}|t-s| \leq\left|t-s^{\prime}\right| \leq \frac{3}{2}|t-s|$, and that we may therefore, by symmetry of the estimate (4.4), assume that $s \geq s^{\prime}$. Whenever $t>s \geq s^{\prime}$, then, by the preceding estimate,

$$
\begin{aligned}
\left|K(t, s)-K\left(t, s^{\prime}\right)\right|_{\mathcal{L}(X)} & =\left|\int_{s^{\prime}}^{s} \frac{\partial}{\partial r} A(t) U(t, r) d r\right|_{\mathcal{L}(X)} \\
& =\left|\int_{s^{\prime}}^{s} A(t) U\left(t, \frac{t+s}{2}\right) \frac{\partial}{\partial r} U\left(\frac{t+s}{2}, r\right) d r\right|_{\mathcal{L}(X)} \\
& \leq\left|A(t) U\left(t, \frac{t+s}{2}\right)\right|_{\mathcal{L}(X)} \int_{s^{\prime}}^{s}\left|\frac{\partial}{\partial r} U\left(\frac{t+s}{2}, r\right)\right|_{\mathcal{L}(X)} d r \\
& \leq 2 C^{2} \frac{1}{t-s} \int_{s^{\prime}}^{s} \frac{1}{\frac{t+s}{2}-r} d r \\
& =2 C^{2} \frac{1}{t-s} \log \left(1+2 \frac{s-s^{\prime}}{t-s}\right) \\
& \leq 4 C^{2} \frac{s-s^{\prime}}{(t-s)^{2}} .
\end{aligned}
$$

Whenever $s \geq s^{\prime}>t$, then

$$
\left|K(t, s)-K\left(t, s^{\prime}\right)\right|_{\mathcal{L}(X)}=0,
$$


by definition of the kernel. The case $s>t>s^{\prime}$ need not be considered since $\left|s-s^{\prime}\right| \leq \frac{1}{2}|t-s|$. Thus, the preceding two estimates imply that $K$ satisfies the second standard condition.

The third condition is a consequence of the estimate in property (vi) and of the uniform exponential stability of the evolution family. Let $t, t^{\prime}, s \in \mathbb{R}$ be such that $\left|t-t^{\prime}\right| \leq \frac{1}{2}|t-s|$. Again, since $\frac{1}{2}|t-s| \leq\left|t^{\prime}-s\right| \leq \frac{3}{2}|t-s|$, and by symmetry of the estimate (4.5), we may assume that $t \geq t^{\prime}$.

If $t \geq t^{\prime}>s$ and $t-s \leq 1$, then the preceding estimate and the estimate from property (vi) imply that for every $\varepsilon>0$ there exists $C_{\varepsilon} \geq 0$ such that

$$
\left|K(t, s)-K\left(t^{\prime}, s\right)\right|_{\mathcal{L}(X)} \leq 2 C_{\varepsilon} \frac{\left(t-t^{\prime}\right)^{\delta}}{\left(t^{\prime}-s\right)^{1+\delta}}
$$

where

$$
\delta=\min \{1,1-\varrho, \alpha, \alpha-\varepsilon, 1-\varepsilon, 1-\varrho-\varepsilon\} .
$$

We may choose $\varepsilon>0$ small enough so that $\delta>0$.

If $t \geq t^{\prime}>s$ and $t-s \geq 1$, we consider two subcases. If $t-t^{\prime} \leq 1$, then we use the preceding estimate and in addition the uniform exponential stability in order to obtain

$\left|K(t, s)-K\left(t^{\prime}, s\right)\right|_{\mathcal{L}(X)}=\left|\left(K(t, t-1)-K\left(t^{\prime}, t-1\right)\right) U(t-1, s)\right|_{\mathcal{L}(X)} \leq C e^{\omega} \sum_{j=1}^{6}\left(t-t^{\prime}\right)^{\delta_{j}} e^{-\omega(t-s)}$.

If $t-t^{\prime} \geq 1$, then we estimate by applying the first standard condition:

$$
\begin{aligned}
\left|K(t, s)-K\left(t^{\prime}, s\right)\right|_{\mathcal{L}(X)} & \leq\left|\left(K\left(t, \frac{t^{\prime}+s}{2}\right)-K\left(t^{\prime}, \frac{t^{\prime}+s}{2}\right)\right) U\left(\frac{t^{\prime}+s}{2}, s\right)\right|_{\mathcal{L}(X)} \\
& \leq \frac{C}{t-s} e^{-\frac{\omega}{2}\left(t^{\prime}-s\right)} \\
& \leq C \frac{t-t^{\prime}}{(t-s)^{2}} .
\end{aligned}
$$

If $s>t \geq t^{\prime}$, then

$$
\left|K(t, s)-K\left(t^{\prime}, s\right)\right|_{\mathcal{L}(X)}=0,
$$

by definition of the kernel. The case $t>s>t^{\prime}$ need not be considered since $\left|t-t^{\prime}\right| \leq \frac{1}{2}|t-s|$. The preceding three estimates imply that $K$ satisfies the third standard condition.

We have proved that $K$ is a standard kernel, and the statement therefore follows from Theorem 4.3.

Remark 7.2. We point out that there is, up to now, no systematic, unified existence theory for the non-autonomous problem (7.1). There are in fact various 
sets of conditions on the operators $A(t)$, different from the Kato-Tanabe conditions I.-II., which lead to the existence of an evolution family $(U(t, s))_{t \geq s \geq 0}$ satisfying the properties (i)-(iv) above. We mention, for example, the AcquistapaceTerreni conditions from [1, p.51] which are independent of the Kato-Tanabe conditions by [1, Theorem 7.9], or the conditions from [41, Theorem 6.1, p.150]. In order to show that the resulting kernel $K(t, s)=A(t) U(t, s)(t>s)$ satisfies the standard conditions, however, some estimates like the estimates in properties (v) and (vi) are helpful. Property (v), including the estimate, is proved for various extended Acquistapace-Terreni conditions, for example if both $(A(t))$ and the adjoint family $\left(A(t)^{*}\right)$ satisfy the Acquistapace-Terreni conditions $[2$, Section 6]. But we do not know whether also property (vi) holds in those cases. On the other hand, under the conditions of [41, Theorem 6.1, p.150], Hölder continuity of $t \mapsto A(t) U(t, s)$ is proved in [41, Theorem 6.9], while the full property (vi) with a similar estimate only follows from the proof (it is tedious but elementary to repeat the proof and keep track of precise estimates of the various terms). But [41] contains no estimate for $\left|\frac{\partial}{\partial s} U(t, s)\right|_{\mathcal{L}(X)}$ like the one in property (v). It is, to our best knowledge, an open question whether all properties (i)-(vi) hold in the case of the extended Acquistapace-Terreni conditions, or in the case of the conditions from [41, Theorem 6.1, p.150].

7.2. The autonomous, first order Cauchy problem on the line. Corollary 7.1 clearly applies to the autonomous problem

$$
\dot{u}+A u=f \quad \text { on } \mathbb{R},
$$

where $A$ is a closed, linear, densely defined operator on a Banach space $X$. If - $A$ generates an exponentially stable $C_{0}$-semigroup $\left(e^{-t A}\right)_{t \geq 0}$, and if the problem (7.2) has $L^{p}$-maximal regularity, then the generated semigroup is necessarily analytic [23]. By the characterization of analytic $C_{0}$-semigroups [41, Theorem 5.2, p.61], the operator $A \equiv A(t)$ therefore satisfies condition I. It is therefore natural to assume condition I. above. Note that in the autonomous problem (7.2) the conditions II. and III. are trivially satisfied. The verification of the properties (i)-(vi) is much easier than in the case of the nonautonomous Cauchy problem; it involves classical results on $C_{0}$-semigroups. As already noted, condition $I$. is equivalent to the fact that $-A$ generates an analytic $C_{0}$-semigroup $(T(t))_{t \geq 0}$, and then $U(t, s)=T(t-s)$ is the associated evolution family (properties (i) and (ii)). Every $C_{0}$-semigroup is exponentially bounded ([41, Theorem 2.2, p.4], property (iii)), and every analytic $C_{0}$-semigroup is infinitely differentiable and the estimate $\left\|A^{k} T(t)\right\| \leq C_{k} / t^{k}$ holds for every $k \geq 0$ and every $t \in(0,1)([41$, Theorem 5.2, p.61], properties (iv), (v) and (vi)). 
Corollary 7.3. Assume that the problem (7.3) on the line has $L^{p}$-maximal regularity for some $p \in(1, \infty)$ and that $-A$ generates an exponentially stable (analytic) $C_{0^{-}}$ semigroup. Then, for every r.i.BFS $\mathbb{E}$ satisfying $1<p_{\mathbb{E}} \leq q_{\mathbb{E}}<\infty$ and every weight $w \in A_{p_{\mathrm{E}}}$ the problem (7.3) has $\mathbb{E}_{w}$-maximal regularity.

7.3. The autonomous, first order Cauchy problem on the interval. The concept of $L^{p}$-maximal regularity or $E_{w}$-maximal regularity is also defined for the autonomous Cauchy problem on a finite interval

$$
\dot{u}+A u=f \quad \text { on }(0, T), \quad u(0)=0 .
$$

We say that this problem has $\mathbb{E}_{w}$-maximal regularity if for every $f \in \mathbb{E}_{w}(0, T ; X)$ there exists a unique strong solution $u \in \mathbb{E}_{w}(0, T ; X)$ such that $\dot{u}$ and $A u$ also belong to $\mathbb{E}_{w}(0, T ; X)$. Here, $\mathbb{E}_{w}(0, T ; X)$ is the space of all functions of the form $u \chi_{(0, T)}$ with $u \in \mathbb{E}_{w}(X)$. Equivalently, by the properties of a function norm, $\mathbb{E}_{w}(0, T ; X)$ is the space of all measurable functions whose extension to $\mathbb{R}$ by 0 belongs to $\mathbb{E}_{w}(X)$.

Corollary 7.4. Assume that the problem (7.3) on the interval has $L^{p}$-maximal regularity for some $p \in(1, \infty)$. Then, for every r.i.BFS $\mathbb{E}$ satisfying $1<p_{\mathbb{E}} \leq q_{\mathbb{E}}<\infty$ and every weight $w \in A_{p_{\mathbb{E}}}$, the problem (7.3) has $\mathbb{E}_{w}$-maximal regularity.

Proof. Note first that the problem (7.3) has $\mathbb{E}_{w}$-maximal regularity for some if and only if for every $\lambda \in \mathbb{R}$ the problem

$$
\dot{v}+A v+\lambda v=g \quad \text { on }(0, T), \quad v(0)=0,
$$

has $\mathbb{E}_{w}$-maximal regularity. In fact, the mappings $u \mapsto v=e^{\lambda \cdot} u$ and $f \mapsto g=e^{\lambda \cdot} f$ show that the two problems (7.3) and (7.4) are similar. By choosing $\lambda \geq 0$ large enough, $-A-\lambda$ is the generator of an exponentially stable, analytic $C_{0^{-}}$ semigroup [23]. Now it is not difficult to show that for a negative generator $A$ of an exponentially stable, analytic $C_{0}$-semigroup, $L^{p}$-maximal regularity of the problem (7.2) on the line and the problem (7.4) on the interval are equivalent. The claim now follows from Corollary 7.3 and by using again the above equivalence of $\mathbb{E}_{w}$-maximal regularity for the problems (7.3) and (7.4).

Remark 7.5. Corollary 7.3 extends the two following extrapolation results from the literature. The first extrapolation result is well known and states that if the Cauchy problem (7.3) has $L^{p}$-maximal regularity for some $p \in(1, \infty)$, then it has $L^{p}$-maximal regularity for every $p \in(1, \infty)$ [45], [13], [29]. However, we also obtain a second extrapolation result [7, Theorem 1.3], [43, Theorem 2.4] which states that $L^{p}$-maximal regularity for some $p \in(1, \infty)(p=2$ in [7]) implies $L_{w_{\theta}}^{p}$-maximal regularity for all $p \in(1, \infty)$ and all power weights $w_{\theta}(\theta \in(0,1))$ of the form

$$
w_{\theta}(s)=|s|^{p-\left(\theta+\frac{1}{p}\right) p} \quad(s \in \mathbb{R}) ;
$$


by Remark 3.2, these weights are special Muckenhoupt weights. In order to be precise, note that in $[43$, Theorem 2.4$] \theta$ ranges in $\left(0,1-\frac{1}{p}\right]$, while we obtain the full range $\theta \in(0,1)$. Theorem 1.3 in [7] contains only the special case when $p=2$ and $X$ is a Hilbert space, but it covers the larger range $\theta \in(0, \infty)$ and thus leaves the class of Muckenhoupt $A_{2}$ weights on $\mathbb{R}$. Note that [7] restricts considerations to the half-line and intervals.

Remark 7.6. Let $\mathbb{E}$ be a r.i.BFS with $1<p_{\mathbb{E}} \leq q_{\mathbb{E}}<\infty$, let $w \in A_{p_{\mathbb{E}}}$ be a Muckenhoupt weight and let $T>0$. Given a linear, closed, densely defined operator $A$ on a Banach space $X$, it is convenient to define the maximal regularity space

$$
M R_{\mathbb{E}_{w}}(0, T ; X, D(A)):=\left\{u \in \mathbb{E}_{w}(0, T ; X): \dot{u}, A u \in \mathbb{E}_{w}(0, T ; X)\right\}
$$

and the associated trace space

$$
\operatorname{Tr}_{\mathbb{E}_{w}}(X, D(A)):=\left\{x \in X: \exists u \in M R_{\mathbb{E}_{w}}(0, T ; X, D(A)) \text { such that } u(0)=x\right\} .
$$

We equip these two spaces with the natural norms

$$
\|u\|_{M R_{\mathbb{E}_{w}}}:=\|u\|_{\mathbb{E}_{w}}+\|\dot{u}\|_{\mathbb{E}_{w}}+\|A u\|_{\mathbb{E}_{w}}
$$

and

$$
\|x\|_{T_{\mathbb{E}_{w}}}:=\inf \left\{\|u\|_{M R_{\mathbb{E}_{w}}}: u \in M R_{\mathbb{E}_{w}}(0, T ; X, D(A)) \text { and } u(0)=x\right\},
$$

so that both spaces are Banach spaces. We leave it as an exercise to show that the trace space does not depend on $T>0$. Both spaces arise naturally in the context of maximal regularity of the linear, first order Cauchy problem on the interval and in the context of interpolation theory. We first observe the following

Lemma 7.7. Assume that the problem (7.3) has $\mathbb{E}_{w}$-maximal regularity. Then, for every $x \in X$ the initial value problem

$$
\dot{u}+A u=0 \quad \text { on }(0, T), \quad u(0)=x,
$$

admits a unique solution $u \in M R_{\mathbb{E}_{w}}(0, T ; X, D(A))$ if and only if $x \in \operatorname{Tr}_{\mathbb{E}_{w}}(X, D(A))$.

Proof. By definition of the trace space, the condition $x \in \operatorname{Tr}_{\mathbb{E}_{w v}}(X, D(A))$ is certainly necessary for existence of a solution $u \in M R_{\mathbb{E}_{w}}(0, T ; X, D(A))$. In order to show sufficiency, let $x \in \operatorname{Tr}_{\mathbb{E}_{w}}(X, D(A))$. Then, by definition, there exists $v \in M R_{\mathbb{E}_{w}}(0, T ; X, D(A))$ such that $v(0)=x$. By definition of the maximal regularity space, $f:=\dot{v}+A v \in \mathbb{E}_{w}(0, T ; X)$. Since the inhomogeneous problem (7.3) has $\mathbb{E}_{w}$-maximal regularity, there exists $w \in M R_{\mathbb{E}_{w}}(0, T ; X, D(A))$ such that $\dot{w}+A w=f, w(0)=0$. Now $u:=v-w$ is a solution of the problem (7.6) and we have proved existence. Uniqueness follows from linearity and uniqueness for the problem (7.3). 
Lemma 7.7 gives precise regularizing effects for solutions of the initial value problem (7.6) in terms of the regularity of the initial value. The link to interpolation theory is clear when we note that for every $p \in(1, \infty)$ and $\theta \in(0,1)$ the equality

$$
\operatorname{Tr}_{L_{w_{\theta}}^{p}}(X, D(A))=(X, D(A))_{\theta, p}
$$

holds [37, Proposition 1.2.10]. Here, the weight $w_{\theta}$ is defined as in (7.5) and the spaces $(X, D(A))_{\theta, p}$ are the classical real interpolation spaces. Summing up, we obtain that if the problem (7.3) has $L^{p}$-maximal regularity for some $p \in(1, \infty)$, then for every $p \in(1, \infty)$, every $\theta \in(0,1)$ and every $x \in(X, D(A))_{\theta, p}$ the initial value problem (7.6) admits a unique solution satisfying $\dot{u}, A u \in L_{w_{\theta}}^{p}(0, T ; X)$.

In principle, we obtain more, since $L^{p}$-maximal regularity for some $p \in(1, \infty)$ implies $\mathbb{E}_{w}$-maximal regularity for every r.i.BFS $\mathbb{E}$ with $1<p_{\mathbb{E}} \leq q_{\mathbb{E}}<\infty$ and every Muckenhoupt weight $w \in A_{p_{\mathrm{E}}}$. The characterization and the properties of the associated trace spaces $\operatorname{Tr}_{\mathbb{E}_{w}}(X, D(A))$ is now certainly an interesting subject, but left as an open problem.

Acknowledgment. We thank the anonymous referee for his/her comments, which contributed to improve the quality of the paper.

\section{REFERENCES}

1. Paolo Acquistapace and Brunello Terreni, A unified approach to abstract linear nonautonomous parabolic equations, Rend. Sem. Mat. Univ. Padova 78 (1987), 47-107.

2. Paolo Acquistapace and Brunello Terreni, Regularity properties of the evolution operator for abstract linear parabolic equations, Differential Integral Equations 5 (1992), no. 5, 1151-1184.

3. H. Amann, Maximal regularity for nonautonomous evolution equations, Advanced Nonlinear Studies 4 (2004), 417-430.

4. Wolfgang Arendt, Semigroups and evolution equations: functional calculus, regularity and kernel estimates, Evolutionary equations. Vol. I, Handb. Differ. Equ., North-Holland, Amsterdam, 2004, pp. 1-85.

5. W. Arendt, R. Chill, S. Fornaro, and C. Poupaud, $L^{p}$-maximal regularity for non-autonomous evolution equations, J. Differential Equations 237 (2007), 1-26.

6. Wolfgang Arendt, Dominik Dier, Hafida Laasri, and El Maati Ouhabaz, Maximal regularity for evolution equations governed by non-autonomous forms, Preprint (2013), arXiv:1303.1167.

7. P. Auscher and A. Axelsson, Remarks on maximal regularity, Parabolic problems, volume 80 of Progr. Nonlinear Differential Equations Appl., pages 45-55. Birkhäuser/Springer Basel AG, Basel, 2011.

8. Jean-Bernard Baillon, Caractère borné de certains générateurs de semi-groupes linéaires dans les espaces de Banach, C. R. Acad. Sci. Paris Sér. A-B 290 (1980), no. 16, A757-A760.

9. A. Benedek, A. P. Calderón, and R. Panzone, Convolution operators on Banach space valued functions, Proc. Nat. Acad. Sci. USA 48 (1962), 356-365.

10. Colin Bennett and Robert Sharpley, Interpolation of operators, Pure and Applied Mathematics, vol. 129, Academic Press Inc., Boston, MA, 1988.

11. J. Bourgain, Some remarks on Banach spaces in which martingale difference sequences are unconditional, Ark. Math. 21 (1983), 163-168. 
12. D. L. Burkholder, A geometric condition that implies the existence of certain singular integrals of Banach-space-valued functions, Conference on harmonic analysis in honor of Antoni Zygmund, Vol. I, II (Chicago, Ill., 1981), Wadsworth Math. Ser., Wadsworth, Belmont, CA, 1983, pp. 270-286.

13. Piermarco Cannarsa and Vincenzo Vespri, On maximal $L^{p}$ regularity for the abstract Cauchy problem, Boll. Un. Mat. Ital. B (6) 5 (1986), no. 1, 165-175.

14. R. R. Coifman and C. Fefferman, Weighted norm inequalities for maximal functions and singular integrals, Studia Math. 51 (1974), 241-250.

15. Ronald R. Coifman and Yves Meyer, Au delà des opérateurs pseudo-différentiels, Astérisque, vol. 57, Société Mathématique de France, Paris, 1978, With an English summary.

16. David V. Cruz-Uribe, José Maria Martell, and Carlos Pérez, Weights, extrapolation and the theory of Rubio de Francia, Operator Theory: Advances and Applications, vol. 215, Birkhäuser/Springer Basel AG, Basel, 2011.

17. Guillermo P. Curbera, José García-Cuerva, José María Martell, and Carlos Pérez, Extrapolation with weights, rearrangement-invariant function spaces, modular inequalities and applications to singular integrals, Adv. Math. 203 (2006), no. 1, 256-318.

18. Michael Ćwikel, Anna Kamińska, Lech Maligranda, and Luboš Pick, Are generalized Lorentz "spaces" really spaces?, Proc. Amer. Math. Soc. 132 (2004), no. 12, 3615-3625 (electronic).

19. G. Da Prato and P. Grisvard, Sommes d'opérateurs linéaires et équations différentielles opérationnelles, J. Math. Pures Appl. (9) 54 (1975), no. 3, 305-387.

20. L. De Simon, Un applicazione della teoria degli integrali singolari allo studio delle equazioni differenziali lineari astratte del primo ordine, Rend. Sem. Mat. Univ. Padova 34 (1964), 547558.

21. R. Denk, M. Hieber, and J. Prüss, R-Boundedness, Fourier Multipliers and Problems of Elliptic and Parabolic Type, Memoirs Amer. Math. Soc., vol. 166, Amer. Math. Soc., Providence, R.I., 2003.

22. Sjoerd Dirksen, Noncommutative Boyd interpolation theorems, Preprint (2012), arXiv:1203.1653.

23. Giovanni Dore, $L^{p}$ regularity for abstract differential equations, Functional analysis and related topics, 1991 (Kyoto), Lecture Notes in Math., vol. 1540, Springer, Berlin, 1993, pp. 25-38.

24. Javier Duoandikoetxea, Fourier analysis, Graduate Studies in Mathematics, vol. 29, American Mathematical Society, Providence, RI, 2001, Translated and revised from the 1995 Spanish original by David Cruz-Uribe.

25. Alberto Fiorenza and Miroslav Krbec, Indices of Orlicz spaces and some applications, Comment. Math. Univ. Carolin. 38 (1997), no. 3, 433-451.

26. Alberto Fiorenza and Miroslav Krbec, A formula for the Boyd indices in Orlicz spaces, Funct. Approx. Comment. Math. 26 (1998), 173-179, Dedicated to Julian Musielak.

27. Bernhard Haak and El Maati Ouhabaz, Maximal regularity for non-autonomous evolution equations, Preprint (2014), arXiv:1402.1136.

28. T. S. Hänninen and T. P. Hytönen, The $A_{2}$ theorem and the local oscillation decomposition for Banach space valued functions, Preprint (2012), arXiv:1210.6236.

29. Matthias Hieber, Operator valued Fourier multipliers, Topics in nonlinear analysis, Progr. Nonlinear Differential Equations Appl., vol. 35, Birkhäuser, Basel, 1999, pp. 363-380.

30. Matthias Hieber and Sylvie Monniaux, Heat kernels and maximal $L^{p}-L^{q}$ estimates: The nonautonomous case, J. Fourier Anal. Appl. 328 (2000), 467-481.

31. Matthias Hieber and Sylvie Monniaux, Pseudo-differential operators and maximal regularity results for non-autonomous parabolic equations, Proc. Amer. Math. Soc. 128 (2000), 1047-1053. 
32. Einar Hille and Ralph S. Phillips, Functional analysis and semi-groups, American Mathematical Society Colloquium Publications, vol. 31, American Mathematical Society, Providence, R. I., 1957, rev. ed.

33. N. J. Kalton and G. Lancien, A solution to the problem of $L^{p}$-maximal regularity, Math. Z. 235 (2000), no. 3, 559-568.

34. Tosio Kato and Hiroki Tanabe, On the abstract evolution equation, Osaka Math. J. 14 (1962), 107-133.

35. Peer C. Kunstmann and Lutz Weis, Maximal $L_{p}$-regularity for parabolic equations, Fourier multiplier theorems and $H^{\infty}$-functional calculus, Functional analytic methods for evolution equations, Lecture Notes in Math., vol. 1855, Springer, Berlin, 2004, pp. 65-311.

36. Stanisław Łojasiewicz, An introduction to the theory of real functions, third ed., A WileyInterscience Publication, John Wiley \& Sons Ltd., Chichester, 1988, With contributions by M. Kosiek, W. Mlak and Z. Opial, Translated from the Polish by G. H. Lawden, Translation edited by A. V. Ferreira.

37. A. Lunardi, Analytic Semigroups and Optimal Regularity in Parabolic Problems, Progress in Nonlinear Differential Equations and Their Applications, vol. 16, Birkhäuser, Basel, 1995.

38. Lech Maligranda, On Hardy's inequality in weighted rearrangement invariant spaces and applications. I, II, Proc. Amer. Math. Soc. 88 (1983), no. 1, 67-74, 75-80.

39. José María Martell, Carlos Pérez, and Rodrigo Trujillo-González, Lack of natural weighted estimates for some singular integral operators, Trans. Amer. Math. Soc. 357 (2005), no. 1, 385-396 (electronic).

40. El Maati Ouhabaz and Chiara Spina, Maximal regularity for non-autonomous Schrödinger type equations, J. Differential Equations 248 (2010), no. 7, 1668-1683.

41. A. Pazy, Semigroups of linear operators and applications to partial differential equations, Applied Mathematical Sciences, vol. 44, Springer-Verlag, New York, 1983.

42. Jan Prüss and Roland Schnaubelt, Solvability and maximal regularity of parabolic evolution equations with coefficients continuous in time, J. Math. Anal. Appl. 256 (2001), 405-430.

43. Jan Prüss and Gieri Simonett, Maximal regularity for evolution equations in weighted $L_{p}$-spaces, Arch. Math. (Basel) 82 (2004), no. 5, 415-431.

44. José L. Rubio de Francia, Factorization theory and $A_{p}$ weights, Amer. J. Math. 106 (1984), no. 3, 533-547.

45. P. E. Sobolevskiü, Coerciveness inequalities for abstract parabolic equations, Dokl. Akad. Nauk SSSR 157 (1964), 52-55.

46. Elias M. Stein, Singular integrals and differentiability properties of functions, Princeton Mathematical Series, No. 30, Princeton University Press, Princeton, N.J., 1970.

47. Elias M. Stein, Harmonic analysis: real-variable methods, orthogonality, and oscillatory integrals, Princeton Mathematical Series, vol. 43, Princeton University Press, Princeton, NJ, 1993, With the assistance of Timothy S. Murphy, Monographs in Harmonic Analysis, III.

48. P. Suryanarayana, The higher order differentiability of solutions of abstract evolution equations, Pacific J. Math. 22 (1967), 543-561.

49. Hiroki Tanabe, Equations of evolution, Monographs and Studies in Mathematics, vol. 6, Pitman (Advanced Publishing Program), Boston, Mass., 1979, Translated from the Japanese by N. Mugibayashi and H. Haneda.

50. William P. Ziemer, Weakly differentiable functions, Graduate Texts in Mathematics, vol. 120, Springer-Verlag, New York, 1989, Sobolev spaces and functions of bounded variation. 
SINGULAR INTEGRAL OPERATORS AND EXTRAPOLATION OF MAXIMAL REGULARITY 35

Institut für Analysis, Fachrichtung Mathematik, Technische Universität Dresden, 01062 DresDen, Germany

E-mail address: ralph.chill@tu-dresden.de

Dipartimento di Architettura - Universitá di Napoli, Via Monteoliveto, 3, I-80134 Napoli, Italy, and Istituto per le Applicazioni del Calcolo "Mauro Picone", sezione di Napoli, Consiglio Nazionale delle Ricerche, via Pietro Castellino, 111, I-80131 Napoli, ITALY

E-mail address: fiorenza@unina.it 